\title{
Explicit filtering and reconstruction turbulence modeling for large-eddy simulation of neutral boundary layer flow
}

\author{
Fotini Katopodes Chow ${ }^{1}$, Robert L. Street ${ }^{1}$, Ming Xue ${ }^{2}$, and Joel H. Ferziger ${ }^{1}$ \\ September 23, 2004
}

1: Environmental Fluid Mechanics Laboratory

Civil and Environmental Engineering, Stanford University

2: School of Meteorology and Center for Analysis and Prediction of Storms

University of Oklahoma

Corresponding author address:

Fotini Katopodes Chow

Atmospheric Science Division

Lawrence Livermore National Laboratory

P.O. Box 808, L-103

Livermore, CA 94551

Phone: 1-925-424-2629, Fax: 1-925-422-5844

katopodes@stanfordalumni.org 


\begin{abstract}
Standard turbulence closures for large-eddy simulations of atmospheric flow based on finite-difference or finite-volume codes use eddy-viscosity models and hence ignore the contribution of the resolved subfilter-scale stresses. These eddy-viscosity closures are unable to produce the expected logarithmic region near the surface in neutral boundary layer flows. Here, explicit filtering and reconstruction are used to improve the representation of the resolvable subfilter-scale (RSFS) stresses, and a dynamic eddy-viscosity model is used for the subgrid-scale (SGS) stresses. Combining reconstruction and eddy-viscosity models yields a sophisticated (and higher-order) version of the well-known mixed model of Bardina et al.; the explicit filtering and reconstruction procedures clearly delineate the contribution of the RSFS and SGS motions. A near-wall stress model is implemented to supplement the turbulence models and account for the stress induced by filtering near a solid boundary as well as the effect of the large grid aspect ratio. Results for neutral boundary layer flow over a rough wall using the combined dynamic reconstruction model and a near-wall stress model show excellent agreement with similarity theory logarithmic velocity profiles, a significant improvement over standard eddy-viscosity closures. Stress profiles also exhibit the expected pattern with increased reconstruction level.
\end{abstract}




\section{Introduction}

In large-eddy simulation (LES) the fluid field is spatially filtered to separate large eddies from smaller motions; the larger scales are simulated accurately, while the effect of the smaller, subfilter scales on the large scales is modeled. The construction of an appropriate subfilter-scale (SFS) turbulence model for LES has been the subject of decades of research. In this paper, we adopt a velocity partitioning approach (Carati et al. 2001) which facilitates the proper modeling of the SFS stresses when finite-difference or finite-volume methods are used. This approach has proven advantageous in low Reynolds number flows (Gullbrand and Chow 2003). We extend the velocity partitioning idea to large-eddy simulation of the atmospheric boundary layer, where the situation is complicated by high Reynolds number flow and a rough lower boundary. The effect of the latter must be dealt with by use of a wall model. This paper focuses on neutrally-stratified flow over flat terrain, but the method can be used for general flow situations.

The definition and application of the LES filters differentiates the velocity partitioning approach from traditional methods. The traditional LES approach treats the grid as the filter which separates large and small motions. The nature of this filter is both unknown, and different, for each term in the equations. The effect of the small motions on the larger scales is modeled as one piece, usually with an eddy-viscosity model. In contrast, the velocity partitioning approach applies a smooth explicit filter (such as a tophat or a Gaussian filter) to separate resolved and SFS motions. It furthermore recognizes that the presence of the numerical grid divides the subfilter-scale (SFS) motions into resolved and unresolved portions. The resolvable subfilter-scale (RSFS) motions can be reconstructed using series expansions, while the unresolvable subfilter-scale (USFS) motions (also called subgrid-scale (SGS)) are modeled separately, typically with an eddy-viscosity model. This method is also known as "explicit filtering and reconstruction" (Gullbrand and Chow 2003). Both the RSFS and SGS models rely on knowledge of the resolved-scale behavior alone, but the RSFS portion can be obtained directly by an inverse filtering operation, as explained in detail later.

Reconstruction of the RSFS stresses requires the definition and application of an explicit filter 
in the LES computation. The filter must be smooth (in wave space) and have a width larger than the grid spacing. The explicit filter is used in the series expansions in the reconstruction procedure and also serves to damp finite-difference and aliasing errors (Lund 1997; Chow and Moin 2003). Thus, explicit filtering and reconstruction are especially useful for finite-volume or finite-difference codes which are easily applied to flows over complex geometries of interest in atmospheric flows. For de-aliased spectral methods, explicit filtering and reconstruction provide no advantage over the traditional approach (see Winckelmans and Jeanmart 2001) because numerical errors are easily controlled.

A well-known problem in atmospheric boundary layer simulations is their lack of agreement with similarity theory in the near-wall region. In this region of the flow, the grid resolution is not adequate to resolve energy-containing scales and the contribution of the SFS model dominates that of the resolved terms (see the discussions in Sullivan et al. (1994); Khanna and Brasseur (1997); Kosović (1997); Juneja and Brasseur (1999)). Standard turbulence closures for atmospheric boundary layer flows use eddy-viscosity models alone (ignoring the contribution of the resolvable subfilter-scale stresses) and do not have correct near-wall behavior. In this paper we explore whether explicit filtering and reconstruction can improve LES results, as demonstrated in low Reynolds number flows (Gullbrand and Chow 2003). The rough bottom boundary in the atmospheric boundary layer requires special treatment (e.g. specifying a log law approximate boundary condition); this will be addressed in the context of velocity partitioning.

We examine a specific test case: the neutral, rotation-influenced, large-scale boundary layer flow considered by Andren et al. (1994). A state-of-the-art atmospheric mesoscale and smallscale simulation model is used, namely, the Advanced Regional Prediction System (ARPS) (Xue et al. 1995, 2000, 2001). ARPS is a finite-difference LES-capable code designed for flow over irregular terrain, so spectral methods and sharp Fourier cutoffs in filters are not viable options. The enhancements to the code are those associated with the new subfilter-scale models (see also Chow and Street (2002) and Chow (2004)). 
Our goal is to learn what logical steps and procedures are required for subfilter-scale modeling to bring the simulated flow fields into agreement with theoretical expectations in the near-wall region. This includes determining appropriate turbulence models and treatments for the rough lower boundary. As a test case for the traditional LES approach, we use the Smagorinsky model (Smagorinsky 1963), which despite its deficiencies, remains commonly used in atmospheric applications. For the explicit filtering and reconstruction approach, we use the approximate deconvolution approach of Stolz and Adams (1999) for the RSFS stresses with the dynamic eddy-viscosity model of Wong and Lilly (1994) to represent SGS motions; these are the components of our dynamic reconstruction model (DRM), similar to that used by Gullbrand and Chow (2003). The properties of the reconstruction model are also discussed by use of the Taylor series expansion model of Katopodes et al. (2000a,b). We also compare results using explicit filtering but ignoring the RSFS term, using the dynamic eddy-viscosity model (Wong and Lilly 1994) alone. Threedimensional filters are used so that our approach is general enough for flow over complex terrain where horizontal planar averages are not applicable. The dynamic eddy-viscosity models require augmentation near the lower boundary, so the near-wall shear stress term of Brown et al. (2001) is included to account for the stress induced by filtering near a solid boundary and for the effect of the large grid aspect ratio typically found near the boundary (Dubrulle et al. 2002; Nakayama and Sakio 2004). This near-wall stress term can be considered part of the SGS model.

Combining reconstruction and eddy-viscosity models yields a generalized (and higher-order) version of the well-known mixed model of Bardina et al. (1983), but now the explicit filtering and reconstruction procedures delineate clearly the contribution of the RSFS and SGS motions. We advocate the general explicit filtering and reconstruction approach. The specific components of the models can vary, and there remains room for much further research, particularly for SGS (including near-wall) models. The RSFS component, as will be shown, can be successfully recovered by series expansion methods. To the authors' knowledge, explicit filtering and reconstruction approaches have not previously been applied to large-scale flows over rough surfaces. 
The following sections summarize the framework for construction of a hybrid, or mixed, LES SFS closure model using separate RSFS and SGS components. We also describe the implementation of the models and results from LES simulations of the neutral boundary layer. The results using the combined DRM and near-wall stress models show excellent agreement with similarity theory logarithmic velocity profiles, which is a significant improvement over standard eddy-viscosity closures.

\section{Decomposition of subfilter-scale stresses}

To facilitate our understanding of the requirements in SFS modeling and especially to improve turbulence models in the near wall region, it is useful to consider velocity partitioning schemes such as those of Carati et al. (2001), Zhou et al. (2001), and Hughes et al. (2001a,b). Figure 1 shows a schematic similar to that of Carati et al. (2001) of a typical energy spectrum from a turbulent flow. The application of a filter (which is smooth in wave space) and a discretization operator (needed to solve the LES equations on a discrete grid) separates the spectrum into three parts. The low wavenumbers are filtered and well resolved on the grid. They are contained in the velocity $\overline{\tilde{u}}_{i}$, where the tilde operator represents the effect of discretization and the overbar an explicit smooth filter. The middle portion (shaded) represents subfilter-scale motions that are between the filter and grid cutoffs and hence resolvable on the grid. These resolved subfilterscale motions can theoretically be reconstructed by an inverse filter operation. Reconstruction is limited, however, by numerical errors (NE) which increase near the grid cutoff due to the modified wavenumber effect on finite-difference evaluation of derivatives (Moin 2001). In addition, there are errors due to aliasing effects, though these are not as important in finite-difference as in spectral codes (Chow and Moin 2003). (Note that the explicit filter damps these numerical errors so that representation of the resolved field is more accurate (Chow and Moin 2003).) The portion to the right of the vertical dashed line contains subgrid-scale motions that cannot be resolved on the grid and must be modeled. These are motions that are smaller than the Nyquist wavenumber cutoff, 
$k_{g}=2 \Delta x$. The amount of energy contained in the SGS portion depends on grid resolution and the distance from the wall (Khanna and Brasseur 1997). In our chosen notation, the tilde operator includes all effects due to numerics (the Nyquist cutoff and numerical errors); we use the symbol loosely because discretization effects are never exactly known. We also emphasize that Fig. 1 is an instantaneous representation of the separation of scales; time evolution will alter the extent to which RSFS motions can be accurately reconstructed, as discussed later.

ARPS employs the spatially filtered compressible nonhydrostatic Navier-Stokes equations. For this paper, ARPS was operated in a quasi-incompressible mode (Xu et al. 1996). Using the notation defined above, the LES governing equations for the resolved velocity are

$$
\begin{gathered}
\frac{\partial \overline{\tilde{u}}_{i}}{\partial t}+\frac{\partial \widetilde{\tilde{\tilde{u}}_{i} \overline{\tilde{u}}_{j}}}{\partial x_{j}}=-\frac{1}{\rho} \frac{\partial \overline{\tilde{p}}}{\partial x_{i}}-g \delta_{i 3}+\epsilon_{i m n} f_{n} \overline{\tilde{u}}_{m}-\frac{\partial \tilde{\tau}_{i j}}{\partial x_{j}} \\
\frac{\partial \overline{\tilde{u}}_{i}}{\partial x_{j}}=0
\end{gathered}
$$

where viscous terms have been neglected. Here $\overline{\tilde{u}}_{i}$ are the velocity components, $\overline{\tilde{p}}$ the pressure, $\rho$ the density, and $f$ the Coriolis parameter. While the discretization effects are different for every term in the equation (due to the various finite-difference schemes used), the same explicit filter is applied to all variables. It is assumed that the filtering and discretization operations commute with the spatial derivatives, which is true for spatially homogeneous filters. Some error is introduced if this is not so (Ghosal and Moin 1995). Further details on the equations used by ARPS are given in Xue et al. (2000) and Chow (2004, Appendices C and D). The configuration used for this work is described further in Section 5a.

We define the total SFS stress as

$$
\tau_{i j}=\overline{u_{i} u_{j}}-\overline{\tilde{u}}_{i} \overline{\tilde{u}}_{j}
$$

Note that when the SFS stress appears in the filtered and discretized Navier-Stokes equations, it appears as $\frac{\partial \tilde{\tau}_{i j}}{\partial x_{j}}$, where the tilde indicates the added effect of the discretization, as in the advection terms. (The discretization operation is essentially a de-aliasing step as well (Chow and Moin 
2003)). The full turbulent stress can be decomposed into resolved and unresolved portions:

$$
\tau_{S F S}=\tau_{i j}=\overline{u_{i} u_{j}}-\overline{\tilde{u}}_{i} \overline{\tilde{u}}_{j}=\underbrace{\left(\overline{u_{i} u_{j}}-\overline{\tilde{u}_{i} \tilde{u}_{j}}\right)}_{\tau_{S G S}}+\underbrace{\left(\overline{\tilde{u}_{i} \tilde{u}_{j}}-\overline{\tilde{u}}_{i} \bar{u}_{j}\right.}_{\tau_{R S F S}})
$$

The first pair of terms on the right-hand side are the subgrid-scale stresses, $\tau_{S G S}$. They depend on scales beyond the resolution domain of the LES and contain the unclosed nonlinear term $\overline{u_{i} u_{j}}$ which must be modeled. The last pair of terms are the filtered-scale stresses, $\tau_{R S F S}$, which depend on the differences between the exact and filtered velocity fields within the resolution domain. This resolved subfilter-scale component, $\tau_{R S F S}$, can theoretically be reconstructed because it is a function of $\tilde{u}_{i}$ which can be obtained by deconvolution (inverse filtering).

Currently available SGS models do not represent the true SGS motions well. It is hoped that by reconstructing the RSFS portion, the overall representation of the SFS stress will be improved, as the SGS contribution will decrease overall. However, near a rough wall, eddy sizes decrease much faster than any grid stretching, and the bulk of the stress contribution comes from SGS terms (Sullivan et al. 2003). In general, the total stress is given by

$$
\tau_{\text {total }}=\tau_{\text {Resolved }}+\tau_{\text {RSFS }}+\tau_{S G S}
$$

where $\tau_{\text {Resolved }}$ is the resolved-scale stress defined in Section $5 \mathrm{c}$ iii. Because eddies scale roughly as distance from the boundary and the filter and grid cutoffs are fixed, as we approach the wall $(z \rightarrow 0)$, it must be that $\tau_{\text {Resolved }} \rightarrow 0$ and $\tau_{R S F S} \rightarrow 0$ because there are no eddies in their respective spectral areas and all of the eddies are subgrid, so $\tau_{\text {total }} \rightarrow \tau_{S G S}$ as $z \rightarrow 0$. The stress on the wall is given by a wall model such as the $\log$ law. Thus $\tau_{S G S}$ supports the total stress at the wall, suggesting the use of a specific near-wall stress model which represents the stress induced by the rough boundary. We employ a separate near-wall stress model as a supplement to the general SGS models used in this work. 


\section{Reconstruction models: series expansion approach}

Using this velocity partitioning framework for the turbulence closure, the RSFS and SGS components can be modeled separately. We first focus on the RSFS components, which can be reconstructed in terms of the resolved velocity. Several methods have been proposed to represent such subfilter-scale motions. Bardina et al. (1983) made a seminal contribution by introducing scale-similarity models, which create an approximation to the full velocity field to estimate the RSFS stress. In Bardina's model, the discrete full velocity is approximated by the filtered velocity, $\tilde{u}_{i} \approx \overline{\tilde{u}}_{i}$, to obtain $\tau_{R S F S} \approx \overline{\overline{\tilde{u}}}_{i} \overline{\tilde{u}}_{j}-\overline{\overline{\tilde{u}}}_{i} \overline{\tilde{\tilde{u}}}_{j}$.

Later models have included those of Yeo and Bedford (1988), Shah and Ferziger (1995), Geurts (1997), Stolz and Adams (1999), Zhou et al. (2001), and Dubrulle et al. (2002) (see the review of Domaradzki and Adams (2002)). All of these methods, except the last two, rely on approximate filter inversion. The method of Zhou et al. (2001) models motions beyond a sharp filter cutoff with an evolution equation; in our context, this approach may be useful as an SGS closure. Dubrulle et al. (2002) also model scales smaller than the grid using a separate dynamic equation. Here, we focus on velocity reconstruction approaches using Taylor series expansions (Katopodes et al. 2000b) and the van Cittert iterative method used in the approximate deconvolution model of Stolz et al. (2001a). These series expansions rely on the application of a smooth explicit filter; a spectral cutoff filter cannot be used.

\section{a. Recursive Taylor series expansions}

Katopodes et al. (2000a,b) used successive inversion of a Taylor series expansion to express the unfiltered (but resolvable) velocity in terms of the filtered velocity. For an isotropic tophat filter applied on a uniform grid, the expansion reduces to

$$
\begin{aligned}
\tilde{u}_{i}(x, y, z) & =\overline{\tilde{u}}_{i}(x, y, z)-\frac{\Delta_{f}^{2}}{24} \nabla^{2} \overline{\tilde{u}}_{i}+\frac{7 \Delta_{f}^{4}}{5760}\left(\frac{\partial^{4} \overline{\tilde{u}}_{i}}{\partial x^{4}}+\frac{\partial^{4} \overline{\tilde{u}}_{i}}{\partial y^{4}}+\frac{\partial^{4} \overline{\tilde{u}}_{i}}{\partial z^{4}}\right) \\
& +\frac{5 \Delta_{f}^{4}}{1728}\left(\frac{\partial^{4} \overline{\tilde{u}}_{i}}{\partial x^{2} \partial y^{2}}+\frac{\partial^{4} \overline{\tilde{u}}_{i}}{\partial y^{2} \partial z^{2}}+\frac{\partial^{4} \overline{\tilde{u}}_{i}}{\partial x^{2} \partial z^{2}}\right)+O\left(\Delta_{f}^{6}\right)
\end{aligned}
$$


to fourth order in the explicit filter width, $\Delta_{f}$. An anisotropic filter is used in simulations, but the isotropic version is shown here for simplicity. Odd powers of $x, y$, or $z$ disappear due to symmetry. Other spatially compact filters give similar results, with a change in the expansion coefficients. The expansion can be extended to an arbitrary order of accuracy by including more terms in the series, though these become cumbersome to compute. Note that the series expansion only recovers scales up to the grid cutoff. The approach can similarly be applied to the scalar transport equation, as done by Katopodes et al. (2000a).

\section{b. Approximate deconvolution method}

High-order reconstruction of the RSFS stress tensor can also be achieved with the iterative deconvolution method of van Cittert (1931). This reconstruction is used by Stolz and Adams (1999) and Stolz et al. (2001a) who call their RSFS model the approximate deconvolution model (ADM). The unfiltered quantities can be derived by a series of successive filtering operations $(G)$ applied to the filtered quantities with

$$
\tilde{u}_{i}=\overline{\tilde{u}}_{i}+(I-G) * \overline{\tilde{u}}_{i}+(I-G) *\left((I-G) * \overline{\tilde{u}}_{i}\right)+\cdots
$$

where $I$ is the identity operator, and $G$ is the explicit filter. This expansion can also be extended to an arbitrary order of accuracy by including more terms in the series, though it is not immediately obvious what the order of magnitude of the next set of terms is. Level- $n$ reconstruction includes the first $n+1$ terms of the series. Computation of higher-order terms is straight-forward, as it simply requires repeated application of the same filter operator.

\section{c. Generation of the $\tau_{R S F S}$ models}

We derive models for $\tau_{i j}$ by substituting a series expansion for the reconstructed velocity $\left(\tilde{u}_{i}^{\star}\right)$ directly into (4) to obtain

$$
\tau_{R S F S}=\overline{\tilde{u}_{i}^{\star} \tilde{u}_{j}^{\star}}-\overline{\tilde{u}_{i}^{\star}} \overline{\tilde{u}_{j}^{\star}}
$$


The series expansions for $\tilde{u}_{i}$ could also be substituted directly into the filtered advection terms of the filtered Navier-Stokes equations, without moving the filtered terms to the right-hand side. This was done by Gullbrand and Chow (2003) and Stolz et al. (2001a), while the SGS contribution was added to the right-hand side as usual. In our implementation, we have both the series expansions and the SGS models on the right-hand side for ease of implementation in ARPS.

For the ADM approach, nothing further is required. In the Taylor series approach, we expand Eq. (8) to derive RSFS models of arbitrary order of accuracy in the (isotropic) filter width, $\Delta_{f}$, giving (to fourth order):

$$
\begin{aligned}
& \tau_{R S F S}=\overline{\overline{\tilde{u}}_{i} \overline{\tilde{u}}_{j}}-\overline{\overline{\tilde{u}}_{i}} \overline{\tilde{\tilde{u}}}_{j}-\frac{\Delta_{f}^{2}}{24} \overline{\overline{\tilde{u}}_{i} \nabla^{2} \overline{\tilde{u}}_{j}}-\frac{\Delta_{f}^{2}}{24} \overline{\overline{\tilde{u}}_{j} \nabla^{2} \overline{\tilde{u}}_{i}} \\
& +\frac{\Delta_{f}^{2}}{24} \overline{\tilde{\tilde{u}}}_{i} \nabla^{2} \overline{\tilde{\tilde{u}}}_{j}+\frac{\Delta_{f}^{2}}{24} \overline{\tilde{u}}_{j} \nabla^{2} \overline{\tilde{\tilde{u}}}_{i}
\end{aligned}
$$

The first two terms are analogous to the Leonard terms in the SFS stress; the higher-order derivative terms can be shown to be dissipative (Clark et al. 1977). To second order in the filter width, equation (9) reduces to the Bardina scale-similarity model, as does the ADM at lowest order.

The series model in Eq. (9) can be rearranged by expanding the higher derivatives and using the filter definition to "unfilter" a few terms. We obtain

$$
\tau_{R S F S}=\frac{\Delta_{f}^{2}}{12} \overline{\frac{\partial \overline{\tilde{u}}_{i}}{\partial x_{m}} \frac{\partial \overline{\tilde{u}}_{j}}{\partial x_{m}}}
$$

which is equivalent to Eq. (9) to fourth order in the filter width (see Katopodes et al. (2000b); Chow (2004) for details). Equation (10) is similar to the model proposed by Clark et al. (1977) (also known as the tensor-diffusivity model, see e.g. Winckelmans et al. (2001)) but with an extra filter. This modified Clark model is considerably simpler than Eq. (9) to implement numerically. We have not, however, presented results for this model here because it can be shown to be equivalent to the ADM (see below) and does not perform better than the ADM, which is easier to implement. The Taylor series method is, however, useful for relating the truncation error to the filter width, which leads to the important property that the series models satisfy the $\tau_{i j}$ evolution equations (see below). (For results using Eq. (10), see Chow (2004); see Winckelmans et al. (2001) and Iliescu 
and Fischer (2003) for other implementations of the tensor-diffusivity model.)

\section{d. Properties of the reconstructed $\tau_{R S F S}$}

A few observations about both reconstruction methods (Eqs. (6) and (7)) are worthwhile. First, neither model has parameters other than the filter width and the number of terms to keep in each series, and no assumptions are made about the form of the RSFS motions. The models should thus be able to capture anisotropic motions. Scale-similar models are also invariant under Galilean transformations (Speziale 1985), and they exhibit correct near-wall behavior in smooth-wall flows when used in a mixed model (Sarghini et al. 1999). Their scale-similarity properties have desirable effects in a priori tests (Katopodes et al. 2000b; Stolz et al. 1999). Furthermore, the evolution equation developed by Katopodes et al. (2000b) for the approximate $\tau_{i j}$ indicates that these resolvable subfilter-scale stresses are influenced by buoyancy, Coriolis, diffusion, pressure, and advection terms, just as the resolved velocities are. Thus the expressions in Eqs. (9) and (10) for $\tau_{i j}$ (and as noted below, the equivalent $\mathrm{ADM}$ expressions) capture the effects of all of the relevant physical mechanisims, to fourth order in the filter width.

While it is not as easy to relate the truncation error of the ADM approach to the filter width, the van Cittert iterative method can easily be related to the the Taylor series reconstruction method. For example, Appendix B of Stolz et al. (2001a) shows how the tensor-diffusivity model can be derived from the ADM. Using finite-difference representations, it is also easy to show the equivalence of these two reconstruction approaches (Chow 2004). A difference in implementation arises when the reconstructed velocities are substituted back into the RSFS stress expressions. In the Taylor series approach, terms of fourth-order or higher are explicitly disregarded, e.g. in the computation of the product terms. That is, we are careful to maintain the order of the final expression even after it has been substituted into $\tau_{i j}$. Discretization is applied only after the final form has been obtained. When the ADM reconstruction terms are used, the discretely reconstructed velocities are substituted directly into the RSFS expression. Therefore, higher-order terms are implicitly 
contained in the RSFS expressions. The difference in the ADM ( $n=1)$ vs. Taylor series (fourth order) approaches is in the fourth order truncation terms. The use of the Taylor series expansions more easily preserves the desired order of the reconstruction, but the ADM approach is much simpler to implement numerically.

In summary, the series expansion representations of the RSFS stresses are theoretically excellent (further discussion can be found in Gullbrand and Chow (2003) and Chow (2004)). They perform well in a priori tests and can be used up to any order of accuracy desired within the grid resolution limitations. The computational cost does increase with increasing levels of reconstruction. Furthermore, interaction with numerical errors in a posteriori tests is not easy to predict; results are examined in the later sections. In the next section, we describe approaches to modeling SGS motions, which become increasingly important at coarse grid resolutions such as those present near the lower surface in atmospheric boundary layer flow simulations.

\section{SGS and wall models}

Aside from the discretization errors in the numerical model and in the reconstruction procedure itself, the above reconstruction methods are exact to within the chosen truncation error. Unfortunately, the turbulence closure problem remains in the SGS terms. Equation (4) still has the unclosed term $\overline{u_{i} u_{j}}$ in the SGS portion of the total SFS stress. The interaction of the SGS and RSFS motions is also hard to predict and may create errors that limit the reconstruction as well. The SGS term must account for any such errors in the RSFS term.

Carati et al. (2001) suggest that there is a separation of scales of sorts between the resolved component and the subgrid component of the velocity field (see Figure 1) that perhaps makes an eddy-viscosity model the best choice for the SGS motions. Unfortunately, they find very poor correlations between eddy-viscosity model quantities and those calculated from direct numerical simulation (DNS) data. For lack of a better framework, a simple eddy-viscosity form is also 
assumed in this work for modeling the unclosed SGS term:

$$
\tau_{S G S}=-2 \nu_{T} \overline{\widetilde{S}}_{i j}
$$

where $\nu_{T}$ is the eddy viscosity, and $\overline{\widetilde{S}}_{i j}=(1 / 2)\left(\partial \overline{\tilde{u}}_{i} / \partial x_{j}+\partial \overline{\tilde{u}}_{j} / \partial x_{i}\right)$ is the resolved strain rate tensor. The closure problem shifts to determining the best representation for $\nu_{T}$. Despite the known shortcomings of this class of models, they are convenient to use when energy transfer to the subgrid scales is desired. The variations of eddy-viscosity models are too numerous to describe them all, so we focus on the models we have chosen for our study. These particular SGS models have been used before to represent the complete SFS stress, but none have been previously used with explicit filtering and reconstruction in large-scale flows. Near the lower boundary, the SGS closure requires special treatment, as described in Section d below.

\section{a. Smagorinsky-based eddy-viscosity models}

One of the most commonly used eddy-viscosity models is the Smagorinsky model (Smagorinsky 1963), which assumes

$$
\nu_{T}=\left(C_{S} \Delta_{g}\right)^{2}\left(2 \overline{\widetilde{S}}_{i j} \overline{\tilde{S}}_{i j}\right)^{1 / 2}
$$

where $C_{S}$ is the Smagorinsky coefficient, and $\Delta_{g}$ is the grid spacing. The Smagorinsky model has several drawbacks, especially near the surface, where it overpredicts the stresses (see the discussion in Juneja and Brasseur 1999). All eddy-viscosity models fail to allow for backscatter of energy from small to large scales. Atmospheric measurements show that backscatter is present near the surface and should be included in LES turbulence closure schemes (see Porté-Agel et al. (2001) and Sullivan et al. (2003)). Correlations from a priori tests show that the eddy-viscosity stress tensors are not aligned with calculations from DNS data (Katopodes et al. 2000b). Furthermore, Mason and Thomson (1992) showed that the failure of the Smagorinsky model near the surface could not be cured by increasing grid resolution. There will always be a region near the rough bottom boundary where the flow is under-resolved. Several modifications and alternatives 
to improve performance near the surface have been proposed (see e.g. Sullivan et al. (1994)), but we use the Smagorinsky model in its standard form to simplify the comparisons.

\section{b. Dynamic eddy-viscosity models}

The standard Smagorinsky model requires the use of a pre-determined coefficient, $C_{S}$. An alternative is the dynamic Smagorinsky model (DSM), where the Smagorinsky coefficient $C_{S}$ is determined automatically by applying the same models at the test filter level (Germano et al. 1991). The assumption is that the same Smagorinsky coefficient could be used if the same equations were applied on a coarser grid. The DSM is widely-used as the eddy-viscosity SGS model in small-scale turbulence studies (see e.g. Germano et al. 1991; Meneveau and Katz 2000). The DSM has been used with explicit filtering in small-scale flows by Winckelmans et al. (2001) and Gullbrand and Chow (2003); this requires special attention to the application of the test filters.

Applications of dynamic models to high Reynolds number flows have been few. Balaras et al. (1995) used the DSM in simulations of high Reynolds number channel flow with rough walls (though still at laboratory scales). Esau (2004) simulated the large-scale neutral atmospheric boundary layer using the DSM and the dynamic mixed model (DMM) of Zang et al. (1993) with some improvement over the standard Smagorinsky model. Porté-Agel et al. (2000) developed a scale-dependent dynamic Smagorinsky model to account for the near-wall region where motions are under-resolved. When applied to a neutral atmospheric boundary layer, they achieved improved logarithmic velocity profiles, but at the cost of an extra filtering step in the dynamic procedure and an empirical function to determine the scale dependence.

Due to sensitivities of the DSM to the bottom boundary condition (see Section $5 \mathrm{~b}$ ), as well as for ease of implementation, we instead use the dynamic model of Wong and Lilly (1994), who simulated the convective boundary layer. Wong and Lilly (1994) present a simplified base model

which requires no calculations of $\bar{S}_{i j}$ during the dynamic procedure. Instead, the base model is 
derived from Kolmogorov scaling, which expresses the eddy viscosity as

$$
\nu_{T}=C^{2 / 3} \Delta_{f}^{4 / 3} \epsilon^{1 / 3}, \quad \text { or } \quad \nu_{T}=C_{\epsilon} \Delta_{f}^{4 / 3}
$$

where $C_{\epsilon}=C^{2 / 3} \epsilon^{1 / 3}$ is the coefficient of interest; hence the traditional requirement that the dissipation rate $\epsilon$ be equal to the SGS energy production rate (including the buoyancy term) is avoided. This base model reduces computational cost because the derivatives in Eq. (12) (at grid and test levels) are no longer required. In the dynamic Wong-Lilly (DWL) formulation, the coefficient is determined using the least-squares method of Lilly (1992):

$$
2 C_{\epsilon} \Delta_{f}^{4 / 3}=\frac{\left\langle L_{i j} \hat{\bar{S}}_{i j}\right\rangle}{\left(1-\left(\Delta_{t} / \Delta_{f}\right)^{4 / 3}\right)\left\langle\hat{\bar{S}}_{i j} \hat{\bar{S}}_{i j}\right\rangle} .
$$

Here, the brackets $<>$ here denote local averaging, ${ }^{-}$denotes the test filter, and

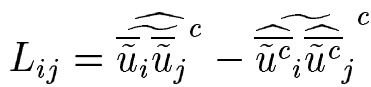

$$
\begin{aligned}
& \widehat{\widehat{\widetilde{S}_{i j}^{c}}}=\frac{1}{2}\left(\frac{\partial{\widehat{\tilde{u}^{c}}}_{i}}{\partial x_{j}}+\frac{\partial{\widehat{\tilde{u^{c}}}}_{j}}{\partial x_{i}}\right) \text {. }
\end{aligned}
$$

We use the notation of Carati et al. (2001) to define the test filtered terms; the ${ }^{\sim c}$ operator denotes the effect of the discretization operator at the coarser test-level grid. The discrete forms of the explicit and test filter operators are given in Eqs. (23-25).

\section{c. Combined RSFS and SGS models}

We use the ADM (for the RSFS) and the DWL (for the SGS) to obtain the total SFS stress:

$$
\tau_{i j}=\overline{\tilde{u}_{i}^{\star} \tilde{u}_{j}^{\star}}-\overline{\tilde{u}_{i}^{\star}} \overline{\tilde{u}_{j}^{\star}}-2 C_{\epsilon} \Delta_{f}^{4 / 3} \overline{\tilde{S}}_{i j}
$$

which we call the dynamic reconstruction model (DRM) (similar to Gullbrand and Chow 2003). We also use ADM reconstruction series from levels zero through ten, denoted DRM-ADM0 through DRM-ADM10. Note that DRM-ADM0 is similar to the DMM with explicit filtering as implemented by Vreman et al. (1994), but with the DWL substituted for the DSM. The standard Smagorinsky model without an RSFS component is our reference case. 
The eddy-viscosity coefficient in this combined RSFS/SGS approach is determined dynamically from

$$
2 C_{\epsilon} \Delta_{f}^{4 / 3}=\frac{\left\langle\left(L_{i j}-H_{i j}\right) \hat{\bar{S}}_{i j}\right\rangle}{\left(1-\left(\Delta_{t} / \Delta_{f}\right)^{4 / 3}\right)\left\langle\hat{\bar{S}}_{i j} \hat{\bar{S}}_{i j}\right\rangle}
$$

where we define (shown here for level-zero reconstruction only)

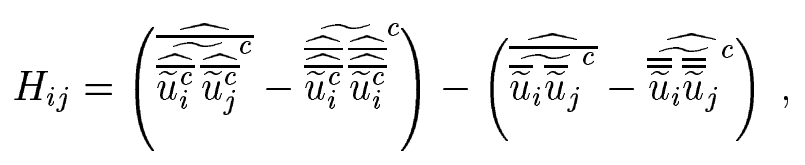

and ask for the reader's indulgence for the use of such compact notation. This derivation again uses the least-squares approach and is similar to that of Zang et al. (1993) and Vreman et al. (1994), thus taking into account the contribution of the scale-similarity portion (in $H_{i j}$ ) while computing the dynamic coefficient. The grid cutoff $\left(\sim, \sim^{c}\right)$ operators are not explicitly applied because no robust means exist for applying a cutoff filter in a finite-difference simulation like ours. The notation serves as a reminder of the effect of the discretization operators. The finite-difference schemes on the coarser grid level limit aliasing effects due to the modified wavenumbers which decay to zero near the grid cutoff. Our combined RSFS and SGS models constitute a mixed model that represents both back-scatter of small-scale energy to the larger scales and forward-scatter (dissipation) of large-scale energy by the small scales. Both processes are essential for reasonable representation of subfilter-scale effects.

Finally, we note that the mixed model (RSFS/SGS) approach could be used with other SGS models, e.g., static Smagorinsky, the modified version of Sullivan et al. (1994), or 1.5-order TKEbased (Deardorff 1980; Moeng 1984) closures commonly used in cloud-scale simulations with parameterizations of stability effects. The values of the turbulent kinetic energy (TKE) equation coefficients are often debated (see e.g. Takemi and Rotunno 2003; Deardorff 1971), so a dynamic procedure like that of Wong and Lilly (1994) is more desirable. Stolz et al. (2001a) use the ADM reconstruction with a relaxation term to drain energy at the smallest scales and also include a dynamic procedure for determining the coefficient. This combination has performed well in simulations of small-scale incompressible channel flows (Stolz et al. 2001a), and compressible flows, e.g. 
shock-turbulent-boundary-layer interaction (Stolz et al. 2001b). For this work, we chose familiar eddy-viscosity formulations for their known performance in large-scale flow simulations.

\section{d. Enhanced near-wall stress model}

Very near the rough lower boundary, the physics of the flow, the grid aspect ratio, and the effect of filtering (in three dimensions) lead to the requirement for special treatment in the turbulence model. In this near-wall region, the eddy size decreases much more rapidly than the grid spacing (Zhou et al. 2001; Sullivan et al. 2003). The vertical grid spacing is invariably smaller than the horizontal one. Scotti et al. (1997) examined the dynamic Smagorinsky model in the context of high-aspect ratio grids where two directions are poorly resolved compared to a third ( $x, y$ compared to $z$ in our case). They suggest that for such grids, higher-wavenumber modes do not have access to all of the local triadic interactions and that this affects the energy spectrum.

Thus, while eddies may be relatively well-resolved in the vertical, they are not so in the horizontal, hence introducing errors. Because $2 \Delta x$ is the minimum eddy size resolved in the horizontal (arguably $4 \Delta x$ is the smallest well-resolved eddy), over a vertical distance of $2 \Delta x$, eddies of this size are still under-resolved. This lack of resolution implies that an additional stress term may be needed near the wall to represent these motions because the chosen SGS models (described above) are inadequate in this very near surface region.

Furthermore, the filtering process and the physical existence of subgrid roughness alter the distribution of stresses near the surface. Dubrulle et al. (2002, see their Eq. (16)) show that the filtering process near a smooth solid boundary generates extra stress terms in the momentum equations near the wall. Nakayama and Sakio (2002) also investigated this filtering process by examining the effects of subgrid roughness with DNS of flow over a wavy bottom boundary consisting of small and large wavelengths. Filtering the DNS flow field and the wavy DNS boundary produced a snapshot of an ideal LES solution over the large wavelength boundary with subgrid roughness. The filtered velocities at the surface in the LES domain were then apparently only influenced by the larger 
wavelength topography, but the subfilter-scale roughness elements (the smaller wavelengths in the original DNS boundary) had generated extra stress near the new, smoother boundary. The surface stress, originally distributed over the DNS boundary, became distributed over a finite vertical layer above the smoother LES boundary. Nakayama and Sakio (2004) and Nakayama et al. (2004) used formal explicit filtering in the neighborhood of the rough wall boundary to derive the extra stresslike terms that appear in the equations. Use of DNS data allowed them to examine the distributions of these terms and to suggest useful ideas to formulate the near-boundary conditions.

The issues of near-wall resolution and physics and of the near-wall filtering point to the need for a near-wall stress model that distributes stresses generated at the rough wall over a region near the wall. Somewhat similar behavior arises in flow through and over vegetation canopies (see e.g. Shaw and Schumann 1992; Patton et al. 2001). The solution in these cases is a canopy model to distribute fluxes over the plant canopy height to account for the increased drag from the vegetation. Brown et al. (2001) extended the idea of plant canopy models to flow over rough surfaces, to distribute stresses generated at the rough surface over a finite distance from the wall.

In accordance with the effects described above, our near-wall stress term is limited to the near wall region, so we model it separately from the main SGS closure. We have implemented the model of Brown et al. (2001), which can be expressed as a forcing term in the horizontal momentum equations, $-C_{c} a(z)|\bar{u}| \bar{u}_{i}$, where $i=1,2$. Here $C_{c}$ is a scaling factor and the function $a(z)$ allows for a smooth decay of the forcing function as the specified cutoff height, $h_{c}$, is approached. $a(z)$ is set equal to $\cos ^{2}\left(\pi z / 2 h_{c}\right)$ for $z<h_{c}$ and is zero otherwise. When implemented numerically, the enhanced stress is included in the turbulence closure stress term, and therefore is integrated numerically using the trapezoidal rule:

$$
\tau_{i, n e a r-w a l l}=-\int C_{c} a(z)|\bar{u}| \bar{u}_{i} d z
$$

where the integration constants are chosen so that $\tau_{i, n e a r-w a l l}=0$ at the top of the enhanced stress layer. This stress is then directly added to the $\tau_{i 3}$ terms from the other model components. The recent work of Nakayama et al. (2004) and the successful simulation of the stable boundary 
layer over flat, rough terrain by Cederwall (2001) suggest that this form is both physically and mathematically reasonable.

\section{Large-eddy simulations of neutral boundary layer flow}

To test the performance of the RSFS/SGS partitioning approach, we use the ARPS code to simulate a rotation-influenced neutral boundary layer flow case similar to that of Andren et al. (1994). For the laminar case, or with a constant eddy viscosity, this flow has the Ekman spiral as an analytical solution (see Stull 1988, pp. 210-212). The fully turbulent solution has been examined by several researchers, e.g., Sullivan et al. (1994), Andren et al. (1994), Kosović (1997), Coleman (1999) (DNS), Porté-Agel et al. (2000) (no Coriolis forcing), Ding et al. (2001), Redelsperger et al. (2001), Carlotti (2002), and Esau (2004). Using scale analysis, Blackadar and Tennekes (1968) showed that the near-wall region of the turbulent Ekman layer should follow a logarithmic law. Traditional eddy-viscosity closures do not give a good logarithmic region near the wall, usually overpredicting the shear in the model with the velocity too low at the wall, and too high further away from it (Andren et al. 1994).

The following sections compare the explicit filtering and reconstruction approach (RSFS plus SGS) with results from the standard Smagorinsky model available in ARPS. The TKE 1.5 closure in ARPS gives results similar to the Smagorinsky model, so they are not shown. The simulation setup and the effects of dynamic reconstruction on the flow solution are described below.

\section{a. Model details and flow setup}

ARPS was developed at the Center for Analysis and Prediction of Storms at the University of Oklahoma. Intended mainly for mesoscale and small-scale atmospheric simulations, ARPS solves the three-dimensional, compressible, non-hydrostatic, filtered Navier-Stokes equations. ARPS is employed here in LES mode by using appropriate turbulence closure models. ARPS details can 
be found in Xue et al. (1995, 2000, 2001). As noted in Section 2, the equations have been slightly modified (as detailed in $\mathrm{Xu}$ et al. 1996) to make them closer to the incompressible case studied by Andren et al. (1994). After this work was completed, we performed tests with the original compressible ARPS code; the results were essentially the same as those for the quasi-incompressible case described here.

The flow is driven by a constant pressure gradient corresponding to a geostrophic wind of $\left(U_{g}, V_{g}\right)=(10,0) \mathrm{m} / \mathrm{s}$. ARPS is run for thirty non-dimensional time periods $t f$, where $f$ is the Coriolis parameter, set equal to $1 \times 10^{-4} \mathrm{~s}^{-1}$. This configuration results in an Ekman-like spiral for the mean velocities. The initial conditions are the analytical Ekman spiral solution with small perturbations that trigger instabilities so that the flow becomes fully turbulent (see Figure 2 later).

The grid size for the control case is $(43,43,43)$ with grid spacings of $32 \mathrm{~m}$ in the horizontal. In ARPS this corresponds to a domain size of $\Delta x(n x-3)=1280 \mathrm{~m}$ in each horizontal direction. In the vertical, a stretched grid is used, with $\Delta z_{\text {min }}=10 \mathrm{~m}$ spacing near the bottom and up to $65 \mathrm{~m}$ near the top of the domain. The average spacing is $37.5 \mathrm{~m}$ and the domain height $(H)$ is $1500 \mathrm{~m}$. Fourth-order spatial differencing was used for the advection terms. Temporal discretization uses a mode-splitting technique to accommodate high-frequency acoustic waves; the large time steps $(0.5 \mathrm{~s})$ use the leapfrog method, while first-order forward-backward explicit time stepping is used for the small time steps $(0.05 \mathrm{~s})$ (except for terms responsible for vertical acoustic propagation, which are treated implicitly). Higher and lower grid resolutions as well as different grid aspect ratios were also studied. Run parameters are given in Table 1 for all the tests and the base case.

The top and bottom boundaries are treated as rigid free-slip (also called semi-slip at the lower boundary). Surface momentum fluxes (e.g. $\left.\overline{u w}\right|_{s}$ ) are parameterized to account for the influence of the rough bottom surface with an instantaneous logarithmic drag law (used here with constant drag coefficients) at each grid point:

$$
\left.\overline{u w}\right|_{s}=C_{D} \overline{\tilde{u}} \sqrt{\overline{\tilde{u}}^{2}+\overline{\tilde{v}}^{2}}
$$

where $\overline{\tilde{u}}$ and $\overline{\tilde{v}}$ are evaluated at the first grid point above the wall (at height $\Delta z_{\min } / 2$ ). The drag 
coefficient $C_{D}$ is determined by

$$
C_{D}=\left[\kappa \ln \left(\frac{z+z_{0}}{z_{0}}\right)\right]^{-2}
$$

where $\kappa$ is the von Kármán constant (0.4) and $z_{0}$ is the bottom roughness height, set to $0.1 \mathrm{~m}$, and again $z$ is set to $\Delta z_{m i n} / 2$. For the high-resolution and low aspect ratio grids used here, it may be useful to use fluctuating surface-flux coefficients (as done by Wyngaard et al. 1998), but they found little improvement from this step because of deficiencies in SGS models in the surface layer. At the lateral boundaries, periodic conditions are used for this idealized flat-terrain study.

\section{b. Implementation of RSFS, SGS, and wall models}

The Smagorinsky closure provides the base standard against which we compare our new turbulence modeling approaches. The Smagorinsky coefficient is chosen as $C_{S}=0.18$ (see e.g. Sullivan et al. 1994).

The anisotropic explicit filter for the reconstruction models was applied at twice the grid spacing, $2 \Delta_{g}$; this choice is consistent with the findings of Ghosal (1996) and Chow and Moin (2003), which dictate that a minimum filter-grid ratio of two should be used with fourth-order advection schemes (as in ARPS). This filter appears in the calculation of the RSFS terms and in the dynamic procedure. For ease of implementation, we apply the van Cittert series expansion reconstruction procedure in computational space which has a uniform grid (though still allowing for anisotropy); therefore the filters are applied in computational space (Jordan 1999; Stolz et al. 2001a). The greatest difficulty in implementation of the RSFS models is near solid boundaries, where care must be taken in representing higher-order terms. The values at the wall can be sensitive to the bottom boundary conditions due to the repeated filtering that occurs there. However, we found that when RSFS models are used together with SGS models at the wall, the RSFS contribution decays to zero (see Section 2) regardless of the specified boundary conditions.

On the other hand, our tests of the dynamic Smagorinsky model (DSM) as the SGS model in 
ARPS showed very strong sensitivity to the bottom boundary conditions. Porté-Agel et al. (2000) also performed tests with the standard DSM and obtained values of the dynamic coefficient that were too low near the wall. The dynamic Wong-Lilly (DWL) model was not as sensitive to the chosen boundary conditions (because $\nu_{T}$ does not depend on $\bar{S}_{13}$ explicitly). The stress provided by the DWL model is still too small at the wall, but the resulting profile is better. In addition to giving more reasonable profiles near the wall, the DWL is easier to implement and more computationally efficient than the DSM. Wong and Lilly (1994) used a horizontal plane average, which is not general enough to apply to flow over terrain; we therefore use a local filter created by applying the test filter twice. The dynamic eddy viscosity is also clipped at $-1.5 \times 10^{-5}$ to prevent the eddy viscosity from becoming too negative locally and causing instabilities (see Zang et al. 1993; Wong and Lilly 1994); further details are in Chow (2004).

The only input parameter required for the DWL is the ratio of the test and explicit filters, $\Delta_{t} / \Delta_{f}$, which is chosen to be two, consistent with the $\Delta_{f} / \Delta_{g}$ ratio chosen above (Gullbrand and Chow 2003). The resulting test filter is thus $4 \Delta x$, which differs from the usual test filter used without explicit filtering. When no reconstruction is used, the test filter is often chosen as twice the grid spacing (see e.g. Zang et al. 1993). The choice of the test filter width is a distinguishing, though subtle, feature of the explicit filtering approach (Winckelmans et al. 2001; Gullbrand and Chow 2003). The resulting test plus explicit filter must be similar in shape to the original explicit filter (Winckelmans et al. 2001). The discrete tophat explicit filter is applied (to the streamwise velocity $u$, for example) with

$$
\bar{u}_{i}=0.25 u_{i-1}+0.5 u_{i}+0.25 u_{i+1}
$$

where $i$ temporarily denotes the grid index; therefore the test filter is

$$
\hat{u}_{i}=0.5 u_{i-1}+0.5 u_{i+1}
$$

to give a combined test plus explicit filter of

$$
\hat{\bar{u}}_{i}=0.125 u_{i-2}+0.25 u_{i-1}+0.25 u_{i}+0.25 u_{i+1}+0.125 u_{i+2},
$$


which is simply a tophat filter of twice the width of the explicit filter (for further details see Gullbrand and Chow 2003).

Following Brown et al. (2001), Cederwall (2001) and Chow and Street (2002), we implement a near-wall stress model, based on the model given previously in Eq. (20). Brown et al. (2001) choose a constant $C_{c}$ so that the velocity at the top of the canopy matches experimental measurements. Cederwall (2001) selected $C_{c}$ such that the near-wall stress model augmented the total stress at the first grid point above the wall to make it equal to the total local bottom shear stress. We allow $C_{c}$ to be locally proportional to the bottom shear stress in each horizontal direction. The proportionality factor is chosen so that the near-wall stress model provides the necessary augmentation that yields logarithmic mean velocity profiles near the wall. The variation of $C_{c}$ and $h_{c}$ (the layer height) with grid spacing and aspect ratio is described later. The near-wall stress model is part of the SGS model component, but is treated separately from the eddy-viscosity contribution because it is present strictly very near the wall.

Finally, current turbulence models often do not provide enough dissipation at the highest frequencies, so computational mixing terms may be added for stability if desired. We follow the recommendations in ARPS and use a small fourth-order term, which can be considered to be a type of hyper-viscosity. ARPS also includes a divergence damping term to control acoustic noise. The impact of these damping terms has been assessed and found to be minimal (see spectra plotted in Chow 2004).

\section{c. Simulation results}

i. Time evolution Figures $2 \mathrm{a}$ and $\mathrm{b}$ show the evolution of the non-stationarity measures $C_{u}$ and $C_{v}$ defined by (following Andren et al. (1994))

$$
\begin{aligned}
C_{u} & =-\frac{f}{\overline{u w}_{s}} \int_{0}^{H}\left(<\overline{\tilde{v}}>-V_{g}\right) d z \\
C_{v} & =\frac{f}{\overline{v w}_{s}} \int_{0}^{H}\left(<\overline{\tilde{u}}>-U_{g}\right) d z
\end{aligned}
$$


where $H$ is the stress-free top of the boundary layer and simulation domain, and the $\overline{u w}_{s}$ and $\overline{v w}_{s}$ denote the total surface stresses in the $u w$ and $v w$ planes, respectively. The brackets $<>$ denote horizontal planar averaging, as well as time averaging, using data taken at each time step. At steady-state, both $C_{u}$ and $C_{v}$ should be unity, but even after $100000 \mathrm{~s}(t f=10)$, inertial oscillations are apparent in Figures $2 \mathrm{a}$ and $\mathrm{b}$. These oscillations have a period of $2 \pi / f$ and result from the change in balance of pressure gradient and Coriolis forces (Cushman-Roisin 1994). Note that the spin-up time is much shorter for the DRM-ADM0 run. Andren et al. (1994) state that the oscillations affect primarily the first-order statistics of the flow, but some of our comparisons are quite sensitive, so we seek a solution as close to a statistically steady state as possible. Unless otherwise indicated, we average from $200000 \mathrm{~s}$ to $300000 \mathrm{~s}(t f=20$ to 30$)$ where oscillations in the $C_{v}$ curve especially have decayed significantly. Our simulation and averaging times are about three times as long as those of Andren et al. (1994).

ii. First-order quantities Figure 3a shows semi-log plots of mean wind speed from four different turbulence model configurations: no model, static Smagorinsky, DWL, and DRM-ADM0. The wind speed $\left(U=\sqrt{\overline{\tilde{u}}^{2}+\overline{\tilde{v}}^{2}}\right)$ is normalized by the (time averaged) friction velocity defined by $u_{*}=$ $\left(\overline{u w}_{s}^{2}+\overline{v w}_{s}^{2}\right)^{1 / 4}$ (found to be approximately 0.44). The theoretical log law profile is also shown. The 'no model' and Smagorinsky results deviate considerably, but the DWL and DRM results provide good agreement. The 'no model' simulation still incorporates the rough wall boundary condition, therefore the surface stress contributes at the first point above the wall and slows the wind speed.

A more sensitive measure of a model's performance is the non-dimensional velocity gradient, $\Phi$, defined as

$$
\Phi=\frac{\kappa z}{u_{*}} \sqrt{\left(\frac{\partial<\overline{\tilde{u}}>}{\partial z}\right)^{2}+\left(\frac{\partial<\bar{v}>}{\partial z}\right)^{2}} .
$$

Here $\kappa$ is the von Kármán constant, chosen to be 0.4 . In a logarithmic region, $\Phi=1$, which we expect for approximately the first $150-250 \mathrm{~m}$ above the wall, or $10-15 \%$ of the boundary layer 
depth $(0.1-0.15 H)$ (Sullivan et al. 1994). Profiles of $\Phi$, shown in Figure 3b, have been smoothed to remove $2 \Delta z$ waves amplified by the derivatives (and not visible in the original velocity fields). We see that the overshoot in $\Phi$ reaches 1.6 for the traditional Smagorinsky model, indicating the model allows excessive shear near the surface. Similar overshoots were observed in other studies (Andren et al. 1994; Sullivan et al. 1994; Kosović 1997). It appears that the Smagorinsky model behaves as a large viscosity model that mimics the molecular viscosity dissipation near a smooth wall. In large-scale flows, however, the linear region is confined to a few millimeters above the surface, and the log region should cover the entire range near the wall in our simulation results. Our efforts at improving near-surface $\Phi$ profiles are aimed at replacing this gradient-diffusion behavior near the wall. Surprisingly, the results using no model appear fairly logarithmic after the first point above the wall, but the velocity magnitude is significantly over-predicted.

When reconstruction and dynamic eddy-viscosity models are used with the near-wall stress term (DRM-ADM0), values of $\Phi$ within 0.1 of the ideal value (unity) are obtained. This represents a significant improvement over the standard Smagorinsky simulations. It is better than several previous attempts to improve $\Phi$ profiles, where agreement within about 0.2 from unity was achieved by Sullivan et al. (1994), the backscatter results of Mason and Brown in Andren et al. (1994), PortéAgel et al. (2000), and Ding et al. (2001), using various SGS methods. Our results are comparable to those of Kosović (1997), where $\Phi$ profiles were within 0.1 of unity. The simulations of Esau (2004) using a dynamic mixed model (with two-dimensional filtering) did not produce satisfactory results near the wall (see Esau (2004), Figs. 11a and 15d); while the DMM provides some reconstruction, it was not used with a formal explicit filter, making the level of reconstruction ambiguous. Sensitivity to the numerical formulation with the Smagorinsky model as the base model for the dynamic procedure (as opposed to DWL) and the absence of a near-wall stress model may also have limited the improvement obtained by the DMM.

Our hybrid SFS model has the advantange that once the filter has been selected, no adjustable parameters are needed aside from those in the enhanced near-wall stress which is active only in 
the first few grid points above the wall. The reconstruction component is of scale-similar form, and thus allows backscatter, i.e., energy flux from the small to the large scales (within the grid resolution). This is believed to be especially important in the atmospheric boundary layer when the large scales are not fully resolved and it aids in achieving a logarithmic mean velocity profile. The influence of the enhanced near-wall stress is felt only near the wall, where it helps to straighten the $\Phi$ profiles. The impact of the near-wall stress model does not overwhelm the near-wall results to the point where the turbulence model becomes irrelevant. Tests with the same level of near-wall stress added to the standard Smagorinsky model (without reconstruction) did not produce encouraging results (see Fig. 4). Interestingly, the DWL model without reconstruction also performs quite well in these first-order comparisons.

Figure 5 shows the effect of increasing the level of reconstruction in the DRM-ADM models. The $\Phi$ profiles indicate that while all the results remain within 0.1 of the ideal value, increasing reconstruction moves the profiles slightly closer to unity very near the wall $(z / H<0.05)$. Because the curves are so close together, there is some sensitivity to the time averaging period; Figs. $2 \mathrm{a}$ and b show the deviations from stationarity differed in time with different turbulence models. We found that taking a long averaging period (100000 s) after $C_{u}$ and $C_{v}$ have decayed significantly gave the most reasonable results. However, it is not clear that $\Phi$ should be the sole parameter used for comparisons as it is quite sensitive to small wiggles which are not readily apparent from the mean velocity profiles in Fig. 3a. The effects of adding reconstruction become clearer when second-order statistics are examined.

iii. Second-order quantities Figure 6 compares the $u w$ stress distribution for the Smagorinsky, DWL, and DRM-ADM0 cases from Fig. 3. The resolved $u w$ stress is defined as $<(\overline{\tilde{u}}-<\overline{\tilde{u}}>$ )$(\overline{\tilde{w}}-<\overline{\tilde{w}}>)>$. The Smagorinsky and DWL simulations show similar contributions of the SFS stress, which is largest near the wall. When reconstruction is added, the total SFS stress increases compared to the eddy-viscosity simulations, while the resolved stress decreases accordingly. Sim- 
ilar behavior is observed for the other stress tensor components. The magnitude of the total stress (resolved and SFS) differs slightly between the different simulations.

The contributions from each SFS stress component for DRM-ADM0 are seen in the $u w$ stress profiles in Figure 7. The profiles are also shown on a log plot to magnify the region near the wall. The total $u w$ stress (resolved plus SFS) is not linear because there is a component in the $v w$ direction due to Coriolis forcing. The influence of the near-wall stress model decreases with height, becoming zero at the $h_{c}=4 \Delta x=128 \mathrm{~m}$ for this grid. This is equivalent to the minimum well-resolved horizontal eddy size beneath the filter. We obtain good results with a near-wall stress model proportionality factor $C_{c}=0.5$, so that the near-wall stress model contributes an amount equal to half the wall stress at the first grid point above the wall. The reconstruction terms (RSFS stress components) decay to zero naturally at the wall in the presence of the other two model components (eddy viscosity and near-wall stress) (see Section 2).

The effects of increasing reconstruction on the $u w$ stresses are shown in Fig. 8. The RSFS component of the SFS stress increases with reconstruction level (levels 0, 1 and 5), while the SGS component decreases slightly. Thus, the total SFS contribution increases with increasing reconstruction (primarily due to the contribution of the reconstruction terms), and the resolved contribution decreases accordingly (as seen in Fig. 6). Note that the spacing between the level0 and level-1 curves is about the same as that between level-1 and level-5, indicating a trend towards convergence. This trend was also observed in the small-scale channel flow simulations of Gullbrand and Chow (2003). The slightly decreasing contribution of the eddy-viscosity term is also apparent in Fig. 9 which shows profiles of $\nu_{T}$ for the same simulations as in Fig. 5. Near the wall, the eddy viscosity is relatively constant with height. This is the region where wall effects in the numerical procedure are strongest and the eddy viscosity needs the augmentation provided by the near-wall stress model.

For these high Reynolds number simulations, we have no DNS result to provide exact values of the $\tau_{13}$ SFS stresses for comparison, as was done by Gullbrand and Chow (2003). The turbulent 
stresses can, however, be compared to finer grid resolution simulations treated as estimates of an exact solution. These estimates can then be used to calculate SFS stress values on the coarser grid by using the definition of the turbulent stress tensor in Eq. (3). The finer grid data come from ARPS simulations of twice the resolution in each direction $(83,83,83)$. The horizontal spacing is $16 \mathrm{~m}$ and the vertical resolution is $5 \mathrm{~m}$ near the wall, and stretched above that to an average of 18.75 m over a domain of the same size as the base case $(43,43,43)$ grid. The discrete quantities $\tilde{u}_{i}$ can be obtained from the instantaneous high-resolution fields a posteriori by using a sharp cutoff filter that matches the coarse grid resolution $(43,43,43)$ simulations, but we do not apply the cutoff filter here for simplicity (and the inability to apply it in the vertical direction); tests on the small-scale channel flow code showed that the cutoff filter applied in the horizontal directions did not change the magnitude or shape of the resulting stress profiles significantly. We have also reconstructed the velocity fields to obtain $\tilde{u}_{i}^{\star}$, which estimates the instantaneous velocity fields from a twice finer grid $(163,163,163)$, though the reconstruction is especially limited near the wall. The high-resolution simulations also require a turbulence model; DRM-ADM0 is used instead of a standard eddyviscosity closure, because it yields good mean velocity quantities (logarithmic near the wall). Thus we calculate $\tilde{\tau}_{i j}=\widetilde{\tilde{u}_{i}^{\star} \tilde{u}_{j}^{\star}}-\widetilde{\widetilde{u}_{i}^{\star} \tilde{\tilde{u}}_{j}^{\star}}$. The explicit filter is applied at twice the $(43,43,43)$ cell spacing (i.e. 4 times the high-resolution spacing); a tophat filter is constructed using the trapezoidal rule for the fine grid fields to match the filter width used in the LES simulations. The same filters are applied in the vertical direction. While this procedure provides only an estimate of the true SFS stresses, at the very least the comparison shows how well we match highly-resolved results (that are much more expensive to compute due to the 16-fold increase in computation time when the reduced time step is taken into account).

The turbulent stresses predicted by the coarse resolution simulations using dynamic reconstruction agree well with the SFS stresses calculated from the finer resolution data. Results from DRM-ADM0 up to DRM-ADM10 are shown in Fig. 10 (without the near-wall stress contribution). The first point above the wall for the fine grid stress is not shown because it cannot be 
calculated accurately; the boundary conditions affect the application of multiple filters (especially in the vertical direction). In fact, the values of the extracted stress within $4 \Delta x$ of the wall are approximate because of the limited resolution available. Stresses predicted by the DWL model (as well as Smagorinsky, not shown) are much too small. Increasing reconstruction on the coarse grid improves agreement with the high-resolution results. This shows that the reconstruction procedure contributes appropriately to the SFS stress, unlike the models without reconstruction, which cannot capture the RSFS motions. Because the reconstruction of the RSFS and the eddy-viscosity representation of the SGS stresses are imperfect, the near-wall stress model is needed to contribute the necessary stress near the wall.

The optimal level of reconstruction is difficult to determine. Clearly, the increase in the RSFS stresses is not linear in the reconstruction level. The series expansion models should converge to a fixed contribution. Figure 10 shows the increase between levels 0 and 1 is much larger than the difference between levels 5 and 10. In the small-scale simulations of Gullbrand and Chow (2003), the improvement between levels 5 and 10 was also not very large. The higher reconstruction levels appear to converge, but the results may still differ from the exact results due to SGS effects. It is also possible that numerical errors interact with the reconstruction procedure to limit our ability to capture all of the RSFS motions. Given the computation costs of reconstruction (see Section v below), it appears that level-2 reconstruction is a good compromise. The increased cost from level 5 to 10 does not seem warranted by the small difference in the results.

While the comparisons of mean velocity and $\Phi$ profiles did not clearly distinguish among the simulations, these comparisons of second-order quantities show that reconstruction of the RSFS stress is important. The DWL results (without reconstruction) also produce good mean velocity profiles (Fig. 3), but cannot achieve correct stress profiles. The stress profiles are especially important in predicting turbulent transport and mixing of scalars, and it is only with the dynamic reconstruction closure models that we are able to obtain accurate representations of these stresses. 
iv. Effects of grid resolution and aspect ratio A robust turbulence model should handle a variety of different grid configurations. Figure 11 shows the effect of doubling the grid resolution on the $\Phi$ profiles for the Smagorinsky and DRM-ADM0 simulations. The high-resolution grid uses twice as many points in each direction over the same domain size to give a horizontal resolution of $16 \mathrm{~m}$ and $\Delta z_{\min }$ of $5 \mathrm{~m}$ at the wall (see Table 1). The near-wall stress model parameters were not changed. The overshoot in the Smagorinsky simulations does not decrease; it simply moves closer to the wall. Similar behavior was observed for eddy-viscosity models used by Mason and Thomson (1992) and Khanna and Brasseur (1997). The DRM-ADM0 show equally good results at both resolutions, giving in this case a somewhat grid-independent result for $\Phi$.

The comparisons in Fig. 11 use a constant aspect ratio of $\Delta x / \Delta z=16 / 5$ near the bottom wall. Larger aspect ratios are common in LES applications where vertical grid refinement is important for capturing the vertical structure of the atmosphere (e.g. stratification). Aspect ratios of $100: 1$ or larger are often used in mesoscale simulations, sometimes with boundary layer parameterizations. Such large aspect ratios distort the minimum resolvable eddies near the wall and place a large burden on the SFS model which must compensate for this. An aspect ratio of unity would be ideal (Kravchenko et al. 1996), but such computations are prohibitively expensive. To better determine the contribution required of the near-wall stress model, we have examined aspect ratios ranging from 1.0 to 12.8 . Larger ratios are not feasible on our small domain and lead to instabilities.

Figures $12 \mathrm{a}$ and $\mathrm{b}$ show the effects of a sample of four different grid spacings or aspect ratios on results using the Smagorinsky and DRM-ADM0 models. The near-wall stress contributions in the DRM-ADM0 simulations have been adjusted to give $\Phi$ profiles that are close to unity (see Table 1). The required contribution of the near-wall stress model changes, as expected, because this model was in part developed to accommodate different grid aspect ratios near the wall.

The two independent near-wall stress layer parameters are the layer height $\left(h_{c}\right)$ and the proportionality factor $\left(C_{c}\right)$. We empirically determined the parameters needed for good results over a 
range of grid sizes and aspect ratios. "Good results" mean the ability to achieve $\Phi$ profiles within 0.2 of unity near the wall. Tests indicate that the aspect ratio is less of a controlling factor for the first parameter, the layer height, than is the horizontal grid spacing. We argued earlier that the under-resolved region at the wall extends between $2 \Delta x$ (minimum resolved eddy size) and $4 \Delta x$ (minimum well-resolved eddy size) from the wall. Most of the simulations (of various grid sizes) give good results using a near-wall stress layer height equal to $4 \Delta x$. Thus, $h_{c}$ is different in the $(83,83,83)$ versus the $(43,43,43)$ grid cases, for example, for the same flow conditions, emphasizing that the near-wall stress model is grid dependent. Some simulations give better results using layer heights of 2 or $3 \Delta x$, as shown in Table 1 . While the near-wall stress layer thickness appears well-determined by this simple rule, there is likely a limiting factor because the enhanced stress-layer at coarse resolutions should not grow to extend beyond the boundary layer depth.

Figure 13 shows the variation of $C_{c}$ with the aspect ratio $\Delta x / \Delta z$. The $\Delta z$ dependence of $C_{c}$ is not strong, but a slightly better collapse of the data is obtained using the aspect ratio as opposed to $\Delta x$. The value of $C_{c}$ cannot exceed unity, where the near-wall stress model would provide a stress at the first point above the wall exactly equal to the local wall stress. The required contribution of the near-wall stress model increases with aspect ratio (and also with $\Delta x$ ), confirming our hypothesis that an enhanced stress layer is necessary to compensate for the under-resolved region near the wall. The need for the near-wall stress layer does not disappear when the aspect ratio is near unity, but its influence is reduced. Numerous tests indicate that small deviations from the values in Fig. 13 also give acceptable results. Therefore, Fig. 13 provides reasonable guidelines. While the near-wall stress model we have implemented provides (with proper adjustment) the necessary supplement to the total stress at the wall, this remains an important topic for future research.

v. Computational cost The reconstruction models as well as the dynamic eddy-viscosity model are straightforward to implement, but the resulting increase in computational cost is not negligible. We performed simple comparisons to determine the computational costs of the new methods, 
though no effort was made to optimize the changes in the turbulence modeling sections of the ARPS code. The base case simulations were performed using 8 processors in distributed memory mode (via MPI) on an IBM SP Power4 system. The computation time tests were performed for 20 $000 \mathrm{~s}$.

Table 2 shows the increased computational cost of various turbulence closures compared to the standard Smagorinsky model. From tests of DWL model with and without the near-wall stress model, we estimate that the near-wall stress model adds approximately $5 \%$ to the total computation time. The reconstruction procedure also adds significantly to the computation time. The increased cost of the turbulence model alone is also shown.

While the increase in cost is considerable, it must be compared with other means of obtaining equally accurate results. If, for example, the resolution were doubled in each direction, the increase in computational cost would be approximately 16 times, assuming the timestep were reduced by a factor of two. Even with this increase in resolution, Fig. 11 showed that the Smagorinsky model continues to overpredict the values of $\Phi$ near the wall. Given this alternative, the increases in computational time of the DRM-ADM approach seems quite reasonable for the significant improvement in the results obtained. A compromise solution might be to use DRM-ADM0 up to DRM-ADM2, with an increase in total computational cost of less than 50\% over standard closure models. Finally, the increased cost of $50 \%$ seems reasonable when compared to the factor of two increase in cost over static Smagorinsky observed by Esau (2004) using the DMM, and the 50\% increase for using DMM over DSM observed by Cui (1999), both in incompressible codes; the increased cost of the turbulence model portion was not reported. Further optimization (in the filtering subroutines especially) can make the code more efficient.

\section{Conclusions}

The near-wall region in atmospheric boundary layer flow simulations is plagued by poor resolution and empirically-based wall models for the bottom stress, in addition to SGS and RSFS turbulence 
modeling errors. This paper presented an approach to turbulence modeling over rough boundaries which incorporates the ideas of explicit filtering, velocity reconstruction, and near-wall stress modeling. The velocity partitioning approach clearly delineates the required contributions from each component. As a test case, we performed simulations of the rotation-influenced neutral boundary layer flow over flat terrain, similar to the case of Andren et al. (1994).

Traditional turbulence closure models fail to produce the expected logarithmic region near the wall. We therefore began with the context provided by Carati et al. (2001) which led to insights about useful turbulence model decompositions. With explicit filtering, the total SFS stresses are separated into RSFS and SGS components; the RSFS portion can be reconstructed, while the SGS portion must be modeled, though its required contribution is reduced because the RSFS stress is included. Even traditional closure models without explicit filtering require reconstruction when finite-difference or finite-volume schemes are used. In these cases, the lack of an explicitly defined filter makes it difficult to determine a method for reconstruction. Explicit filtering is also important for reducing numerical errors from finite-difference schemes. Near the wall, the SGS contribution requires enhancement by a near-wall stress model to account for the effects of poor resolution, high grid-aspect ratios, and filter-induced stresses at solid boundaries (Nakayama and Sakio 2004; Dubrulle et al. 2002). This framework aids understanding of the required contributions to subfilter-scale stresses, and simulation results show excellent agreement with similarity theory in the logarithmic region.

Two specific alternatives were presented for velocity reconstruction: the Taylor series expansion method of Katopodes et al. (2000a) and Chow and Street (2002), and the approximate deconvolution method of Stolz and Adams (1999). The methods are equivalent within the order of accuracy of the expansions. Truncation error from the Taylor series approach is kept to the desired order of the filter width by explicitly neglecting higher-order terms in the reconstruction of $\tau_{i j}$. The ADM procedure includes higher-order quantities in $\tau_{i j}$, so the truncation error is less well defined, but ADM provides a simple iterative method for calculation of higher-order series expansions for 
reconstruction, so it is used for the neutral boundary layer simulations in this work. Both methods reduce to the scale-similarity model of Bardina et al. (1983) at lowest order and provide backscatter of energy from small to large scales. Both models also satisfy the full $\tau_{i j}$ evolution equations to a pre-defined order of accuracy (though this is clearer when higher-order terms are neglected as done when using the Taylor series approach).

While the series expansion approaches can be used for the RSFS component of the SFS stresses, the SGS component must be modeled separately. We implemented the dynamic eddyviscosity procedure of Wong and Lilly (1994) which requires only the test filter width as a free parameter. The dynamic Wong-Lilly model has been used to provide necessary dissipation, without incurring the expected drawbacks of an eddy-viscosity model, because it is used in conjunction with a scale-similarity model. To enhance the near-wall stress, we use a model based on the canopy stress model of Brown et al. (2001).

The combinations of the ADM reconstruction, the DWL, and the near-wall stress model significantly improved first- and second-order statistics. Mean velocity profiles and non-dimensional shear profiles $(\Phi)$ showed excellent agreement with similarity theory in the logarithmic region. Examination of second-order statistics, particularly the $u w$ stress decomposition, revealed that increasing reconstruction increases the contribution of the RSFS as well as the total SFS stresses, while correspondingly reducing the resolved $u w$ stresses. This increase in the SFS stress clearly distinguishes the DRM-ADM models from the DWL and Smagorinsky results without reconstruction. Using finer resolution data to reconstruct a high-resolution velocity field for comparison, we showed that the increased SFS stresses (from simulations at the base case low resolution) approach the required SFS stress predicted by the finer resolution data. The DWL and Smagorinsky results strongly underpredict stresses. These observations are in agreement with those of Gullbrand and Chow (2003) who showed that the stresses from small-scale channel flows using the DRM approached the predicted stresses from DNS data. Accurate prediction of stresses is especially important for atmospheric flows, where turbulent transport and mixing of scalars such as temperature, 
moisture, aerosols, and pollutants are of interest.

These results for high Reynolds number atmospheric flow simulations over rough boundaries using explicit filtering and reconstruction are very encouraging. Several different grid resolutions and grid aspect ratios have been tested, and reasonable results have been obtained by accounting for the required variation in the near-wall stress model as a function of horizontal grid spacing and grid aspect ratio. We found that the near-wall stress model alone is insufficient for correcting large-eddy simulations near the lower boundary; it must be used in conjunction with a sophisticated explicit filtering and reconstruction approach so that it provides only the necessary near-wall SGS stress. The improvements obtained from the near-wall stress model point to the need for more sophisticated SGS closures that account for the effects of a rough boundary at the lower wall.

In summary, the steps required to achieve improved neutral boundary layer simulations are as follows. First, an explicit filter width must be chosen which is compatible with the discretization scheme used in the code (Chow and Moin 2003; Ghosal 1996). We chose the explicit filter to be twice the grid size, in agreement with the fourth-order central differencing used. The explicit filter is the basis for the reconstruction procedure, which is easiest to implement using the ADM approach. Level-0 reconstruction (DRM-ADM0) significantly improves the mean quantities, and higher-order reconstruction further improves the representation of the SFS stresses especially. The reconstruction approach is used with a dynamic eddy-viscosity procedure which requires a test filter, typically taken to be twice the explicit filter. In our case, the test filter width was four times the grid spacing. The test filter is used to calculate RSFS and SGS contributions at the test level; they are then compared to their values at the explicit filter level to determine the appropriate eddy-viscosity coefficient. Finally, the SGS contribution from the dynamic eddy-viscosity model is not sufficient near the wall, so a separate near-wall stress model is added to enhance the stress. The thickness of this near-wall stress layer is typically chosen to be between 2 and $4 \Delta x$. The proportionality factor which determines the contribution of the enhanced near-wall stresses varies between 0.4 and 0.9 , depending primarily on the grid aspect ratio. Thus, the combined RSFS, SGS 
and near-wall stress approach requires two parameters for the enhanced near-wall stress, plus $a$ priori selection of the explicit and test filter widths.

As LES is applied to problems where more of the energy of the flow is unresolved, the accuracy of the SFS model becomes increasingly important. Although there is increased computational cost, the explicit filtering and RSFS approach is warranted by the resulting improvements in the simulation results. To our knowledge, we have demonstrated for the first time that scale-similarity or reconstruction models used with explicit filtering can dramatically improve results for high Reynolds number, rough, atmospheric boundary layer flows. While we have selected specific RSFS and SGS model components, the velocity partitioning approach can be used with other, simpler SGS closures (e.g. non-dynamic eddy-viscosity models) to improve simulation results. A low-order RSFS component can easily be added to any code with zero-order reconstruction (the Bardina term); this will improve total SFS stress representations when the RSFS component is used with an existing eddy-viscosity model. Further research is required to determine optimal SGS and near-wall stress representations, and the RSFS/SGS approach should also be tested for other flow conditions. The RSFS component (obtained by series expansions) can remain unchanged, but the SGS model may need adjustment depending on the flow and grid resolution. Preliminary tests on moderately convective boundary layer (Chow 2004, Appendix F) and moderately stable boundary layer simulations also show improved agreement with similarity theory.

Acknowledgments The support of a National Defense Science and Engineering Graduate fellowship [FKC] and NSF Grants ATM-0073395 (Physical Meteorology Program: W.A. Cooper, Program Director) [FKC and RLS], ATM-9909007 and 0129892 [MX] are gratefully acknowledged. Acknowledgment is also made to the National Center for Atmospheric Research, which is sponsored by NSF, for the computing time used in this research. Part of this work was performed under the auspices of the U.S. Department of Energy by the University of California, Lawrence Livermore National Laboratory under Contract W-7405-Eng-48. 


\section{References}

Andren, A., A. R. Brown, J. Graf, P. J. Mason, C.-H. Moeng, F. T. M. Nieuwstadt, and U. Schumann, 1994: Large-eddy simulation of a neutrally stratified boundary layer: A comparison of four computer codes. Q. J. R. Meteorol. Soc., 120, 1457-1484.

Balaras, E., C. Benocci, and U. Piomelli, 1995: Finite-difference computations of high Reynolds number flows using the dynamic subgrid-scale model. Theor. Comput. Fluid Dyn., 7, 207-16.

Bardina, J., J. H. Ferziger, and W. C. Reynolds, 1983: Improved turbulence models based on large eddy simulation of homogeneous, incompressible, turbulent flows. Technical Report TF19, Department of Mechanical Engineering, Stanford University, Stanford, California.

Blackadar, A. K. and H. Tennekes, 1968: Asymptotic similarity in neutral barotropic planetary boundary layers. J. Atmos. Sci., 25, 1015-1020.

Brown, A. R., J. M. Hobson, and N. Wood, 2001: Large-eddy simulation of neutral turbulent flow over rough sinusoidal ridges. Bound.-Layer Meteor., 98, 411-441.

Carati, D., G. S. Winckelmans, and H. Jeanmart, 2001: On the modelling of the subgrid-scale and filtered-scale stress tensors in large-eddy simulation. J. Fluid Mech., 441, 119-138.

Carlotti, P., 2002: Two-point properties of atmospheric turbulence very close to the ground: Comparison of a high resolution LES with theoretical models. Bound.-Layer Meteor, 104, 381-410.

Cederwall, R. T., 2001: Large-eddy simulation of the evolving stable boundary layer over flat terrain. Ph.D. dissertation, Stanford University.

Chow, F. K., 2004: Subfilter-scale turbulence modeling for large-eddy simulation of the atmospheric boundary layer over complex terrain. Ph.D. dissertation, Stanford University.

Chow, F. K. and P. Moin, 2003: A further study of numerical errors in large-eddy simulations. J. Comput. Phys., 184, $366-380$. 
Chow, F. K. and R. L. Street, 2002: Modeling unresolved motions in LES of field-scale flows. Amer. Meteor. Soc., 15th Symp. Bound. Layers Turb., 432-435.

Clark, R. A., J. H. Ferziger, and W. C. Reynolds, 1977: Evaluation of subgrid-scale turbulence models using a fully simulated turbulent flow. Technical Report TF-9, Department of Mechanical Engineering, Stanford University, Stanford, California.

Coleman, G. N., 1999: Similarity statistics from a direct numerical simulation of the neutrally stratified planetary boundary layer. J. Atmos. Sci., 56, $891-900$.

Cui, A., 1999: On the parallel computation of turbulent rotating stratified flows. Ph.D. dissertation, Stanford University.

Cushman-Roisin, B., 1994: Introduction to geophysical fluid dynamics. Prentice Hall, Englewood Cliffs, New Jersey, 320 pp.

Deardorff, J. W., 1971: On the magnitude of the subgrid scale eddy coefficient. J. Comput. Phys., $7,120-33$.

- 1980: Stratocumulus-capped mixed layers derived from a 3-dimensional model. Bound.-Layer Meteor., 18, 495 - 527.

Ding, F., S. P. Arya, and Y.-L. Lin, 2001: Large-eddy simulations of the atmospheric boundary layer using a new subgrid-scale model. Part I: Slightly unstable and neutral cases. Environ. Fluid Mech., 1, 29-47.

Domaradzki, J. A. and N. A. Adams, 2002: Direct modelling of subgrid scales of turbulence in large eddy simulations - art. no. 024. J. Turb., 3.

Dubrulle, B., J. P. Laval, P. P. Sullivan, and J. Werne, 2002: A new dynamical subgrid model for the planetary surface layer. Part I: The model and a priori tests. J. Atmos. Sci., 59, 861 - 876. 
Esau, I., 2004: Simulation of Ekman boundary layers by large eddy model with dynamic mixed subfilter closure. Environ. Fluid Mech., 4, 273 - 303.

Germano, M., U. Piomelli, P. Moin, and W. H. Cabot, 1991: A dynamic subgrid-scale eddy viscosity model. Phys. Fluids, 3, 1760-1765.

Geurts, B. J., 1997: Inverse modeling for large-eddy simulation. Phys. Fluids, 9, 3585-3587.

Ghosal, S., 1996: An analysis of numerical errors in large-eddy simulations of turbulence. J. Comput. Phys., 125, 187-206.

Ghosal, S. and P. Moin, 1995: The basic equations for the large eddy simulation of turbulent flows in complex geometry. J. Comput. Phys., 118, 24-37.

Gullbrand, J. and F. K. Chow, 2003: The effect of numerical errors and turbulence models in largeeddy simulations of channel flow, with and without explicit filtering. J. Fluid Mech., 495, 323 341.

Hughes, T. J. R., L. Mazzei, A. A. Oberai, and A. A. Wray, 2001a: The multiscale formulation of large eddy simulation: Decay of homogeneous isotropic turbulence. Phys. Fluids, 13, 505-512.

Hughes, T. J. R., A. A. Oberai, and L. Mazzei, 2001b: Large eddy simulation of turbulent channel flows by the variational multiscale method. Phys. Fluids, 13, 1784-1799.

Iliescu, T. and P. F. Fischer, 2003: Large eddy simulation of turbulent channel flows by the rational large eddy simulation model. Phys. Fluids, 15, 3036 - 3047.

Jordan, S. A., 1999: A large-eddy simulation methodology in generalized curvilinear coordinates. J. Comput. Phys., 148, $322-340$.

Juneja, A. and J. G. Brasseur, 1999: Characteristics of subgrid-resolved-scale dynamics in anistropic turbulence, with application to rough-wall boundary layers. Phys. Fluids, 11, 30543068. 
Katopodes, F. V., R. L. Street, and J. H. Ferziger, 2000a: Subfilter-scale scalar transport for largeeddy simulation. 14th Symposium on Boundary Layers and Turbulence, American Meteorological Society, 472-475.

- 2000b: A theory for the subfilter-scale model in large-eddy simulation. Technical Report 2000K1, Environmental Fluid Mechanics Laboratory, Stanford University.

Khanna, S. and J. G. Brasseur, 1997: Analysis of monin-obukhov similarity from large-eddy simulation. J. Fluid Mech., 251-86.

Kosović, B., 1997: Subgrid-scale modelling for the large-eddy simulation of high-Reynoldsnumber boundary layers. J. Fluid Mech., 336, 151-182.

Kravchenko, A. G., P. Moin, and R. Moser, 1996: Zonal embedded grids for numerical simulations of wall-bounded turbulent flows. J. Comput. Phys., 127, 412-423.

Lilly, D. K., 1992: A proposed modification of the Germano subgrid-scale closure method. Phys. Fluids, 4, 633-635.

Lund, T. S., 1997: On the use of discrete filters for large eddy simulation. Ann. Res. Briefs, Center for Turbulence Research, NASA Ames - Stanford University, 83-95.

Mason, P. J. and D. J. Thomson, 1992: Stochastic backscatter in large-eddy simulations of boundary layers. J. Fluid Mech., 242, 51-78.

Meneveau, C. and J. Katz, 2000: Scale-invariance and turbulence models for large-eddy simulation. Ann. Rev. Fluid Mech., 32, 1 - 32.

Moeng, C.-H., 1984: A large-eddy-simulation model for the study of planetary boundary-layer turbulence. J. Atmos. Sci., 41, 2052-2062.

Moin, P., 2001: Fundamentals of engineering numerical analysis. Cambridge University Press. 
Nakayama, A., K. Hori, and R. L. Street, 2004: Filtering and large eddy simulation of flow over irregular rough surface. Proceedings of the 2004 Summer Program, Center for Turbulence Research, NASA Ames - Stanford University, in press.

Nakayama, A. and K. Sakio, 2002: Simulation of flows over wavy rough boundaries. Ann. Res. Briefs, Center for Turbulence Research, NASA Ames - Stanford University, 313-324.

- 2004: Filtering and large eddy simulation of turbulent flow over complex boundary. Ann. J. of Hydr. Eng., JSCE, 1237-1242.

Patton, E. G., K. J. Davis, M. C. Barth, and P. P. Sullivan, 2001: Decaying scalars emitted by a forest canopy: a numerical study. Bound.-Layer Meteor, 100, 91 - 129.

Porté-Agel, F., C. Meneveau, and M. B. Parlange, 2000: A scale-dependent dynamic model for large-eddy simulation: application to a neutral atmospheric boundary layer. J. Fluid Mech., 415, 261-284.

Porté-Agel, F., M. B. Parlange, C. Meneveau, and W. E. Eichinger, 2001: A priori field study of the subgrid-scale heat fluxes and dissipation in the atmospheric surface layer. J. Atmos. Sci., 58, $2673-2698$.

Redelsperger, J. L., F. Mahe, and P. Carlotti, 2001: A simple and general subgrid model suitable both for surface layer and free-stream turbulence. Bound.-Layer Meteor., 101, 375 - 408.

Sarghini, F., U. Piomelli, and E. Balaras, 1999: Scale-similar models for large-eddy simulations. Phys. Fluids, 11, 1596-1607.

Scotti, A., C. Meneveau, and M. Fatica, 1997: Dynamic Smagorinsky model on anisotropic grids. Phys. Fluids, 9, 1856-1858.

Shah, K. B. and J. H. Ferziger, 1995: A new non-eddy viscosity subgrid-scale model and its 
application to channel flow. Ann. Res. Briefs, Center for Turbulence Research, NASA AmesStanford University, 73-90.

Shaw, R. H. and U. Schumann, 1992: Large-eddy simulation of turbulent-flow above and within a forest. Bound.-Layer Meteor., 61, 47 - 64.

Smagorinsky, J., 1963: General circulation experiments with the primitive equations. Mon. Wea. Rev., 91, 99-152.

Speziale, C. G., 1985: Galilean invariance of subgrid-scale stress models in the large-eddy simulation of turbulence. J. Fluid Mech., 156, 55-62.

Stolz, S. and N. A. Adams, 1999: An approximate deconvolution procedure for large-eddy simulation. Phys. Fluids, 11, 1699-1701.

Stolz, S., N. A. Adams, and L. Kleiser, 1999: Analysis of subgrid scales and subgrid scale modeling for shock-boundary-layer interaction. First International Symposium on Turbulence and Shear Flow Phenomena, 881-886.

- 2001a: An approximate deconvolution model for large-eddy simulation with application to incompressible wall-bounded flows. Phys. Fluids, 13, 997-1015.

— 2001b: The approximate deconvolution model for large-eddy simulations of compressible flows and its application to shock-turbulent-boundary-layer interaction. Phys. Fluids, 13, 2985-3001.

Stull, R. B., 1988: An introduction to boundary layer meteorology. Kluwer Academic Publishers, Boston.

Sullivan, P. P., T. W. Horst, D. H. Lenschow, C. H. Moeng, and J. C. Weil, 2003: Structure of subfilter-scale fluxes in the atmospheric surface layer with application to large-eddy simulation modelling. J. Fluid Mech., 482, 101 - 139. 
Sullivan, P. P., J. C. McWilliams, and C.-H. Moeng, 1994: A subgrid-scale model for large-eddy simulation of planetary boundary-layer flows. Bound.-Layer Meteor., 71, 247-76.

Takemi, T. and R. Rotunno, 2003: The effects of subgrid model mixing and numerical filtering in simulations of mesoscale cloud systems. Mon. Wea. Rev., 131, $2085-2101$.

van Cittert, P., 1931: Zum Einfluß der Spaltbreite auf die Intensitätsverteilung in Spektrallinien II. Zeitschrift für Physik, 69, 298-308.

Vreman, B., B. Geurts, and H. Kuerten, 1994: On the formulation of the dynamic mixed subgridscale model. Phys. Fluids, 6, 4057 - 4059.

Winckelmans, G. S. and H. Jeanmart: 2001, Assessment of some models for LES without/with explicit filtering. Direct and Large-Eddy Simulation IV, Geurts, Friedrich, and Métais, eds., Kluwer, 55-66.

Winckelmans, G. S., A. A. Wray, O. V. Vasilyev, and H. Jeanmart, 2001: Explicit-filtering largeeddy simulation using the tensor-diffusivity model supplemented by a dynamic smagorinsky term. Phys. Fluids, 13, 1385-1403.

Wong, V. C. and D. K. Lilly, 1994: A comparison of two dynamic subgrid closure methods for turbulent thermal-convection. Phys. Fluids, 6, 1016 - 1023.

Wyngaard, J. C., L. J. Peltier, and S. Khanna, 1998: LES in the surface layer: Surface fluxes, scaling, and SGS modeling. J. Atmos. Sci., 55, 1733-1754.

Xu, Q., M. Xue, and K. K. Droegemeier, 1996: Numerical simulations of density currents in sheared environments within a vertically confined channel. J. Atmos. Sci., 53, 770-786.

Xue, M., K. K. Droegemeier, and V. Wong, 2000: The Advanced Regional Prediction System (ARPS): A multi-scale nonhydrostatic atmospheric simulation and prediction model. Part I: Model dynamics and verification. Meteor. Atmos. Phys., 75, 161-193. 
Xue, M., K. K. Droegemeier, V. Wong, A. Shapiro, and K. Brewster, 1995: ARPS Version 4.0 User's Guide. Center for Analysis and Prediction of Storms, University of Oklahoma, Norman, OK.

Xue, M., K. K. Droegemeier, V. Wong, A. Shapiro, K. Brewster, F. Carr, D. Weber, Y. Liu, and D. Wang, 2001: The Advanced Regional Prediction System (ARPS): A multi-scale nonhydrostatic atmospheric simulation and prediction tool. Part II: Model physics and applications. Meteor. Atmos. Phys., 76, 143-165.

Yeo, W. K. and K. W. Bedford: 1988, Closure-free turbulence modeling based upon a conjunctive higher order averaging procedure. Computational methods in flow analysis, Niki and Kawahara, eds., Okayama University of Science, Okayama, 844-851.

Zang, Y., R. L. Street, and J. R. Koseff, 1993: A dynamic mixed subgrid-scale model and its application to turbulent recirculating flows. Phys. Fluids, 5, 3186-3196.

Zhou, Y., J. G. Brasseur, and A. Juneja, 2001: A resolvable subfilter-scale model specific to largeeddy simulation of under-resolved turbulence. Phys. Fluids, 13, 2602-2610. 


\section{List of Tables}

1 List of simulations and parameters. Standard grid sizes are followed by those with various grid aspect ratios. ${ }^{*}$ denotes the base case. . . . . . . . . . . . . . 45

2 Computational cost of different turbulence models compared to the Smagorinsky model. ............................ . . 46 
Table 1: List of simulations and parameters. Standard grid sizes are followed by those with various grid aspect ratios. $*$ denotes the base case.

\begin{tabular}{cccccccc}
\hline \hline Run name & Grid size & $\Delta x$ & $\Delta z_{\min }$ & Large $\Delta t$ & Small $\Delta t$ & $C_{c}$ & $h_{c}$ \\
\hline Smagorinsky* & $(43,43,43)$ & $32 \mathrm{~m}$ & $10 \mathrm{~m}$ & $0.5 \mathrm{~s}$ & $0.05 \mathrm{~s}$ & - & - \\
DWL & $(43,43,43)$ & $32 \mathrm{~m}$ & $10 \mathrm{~m}$ & $0.5 \mathrm{~s}$ & $0.05 \mathrm{~s}$ & 0.5 & $4 \Delta x$ \\
DRM-ADM0 & $(43,43,43)$ & $32 \mathrm{~m}$ & $10 \mathrm{~m}$ & $0.5 \mathrm{~s}$ & $0.05 \mathrm{~s}$ & 0.5 & $4 \Delta x$ \\
DRM-ADM1 & $(43,43,43)$ & $32 \mathrm{~m}$ & $10 \mathrm{~m}$ & $0.5 \mathrm{~s}$ & $0.05 \mathrm{~s}$ & 0.5 & $4 \Delta x$ \\
DRM-ADM2 & $(43,43,43)$ & $32 \mathrm{~m}$ & $10 \mathrm{~m}$ & $0.5 \mathrm{~s}$ & $0.05 \mathrm{~s}$ & 0.5 & $4 \Delta x$ \\
DRM-ADM5 & $(43,43,43)$ & $32 \mathrm{~m}$ & $10 \mathrm{~m}$ & $0.5 \mathrm{~s}$ & $0.05 \mathrm{~s}$ & 0.5 & $4 \Delta x$ \\
DRM-ADM10 & $(43,43,43)$ & $32 \mathrm{~m}$ & $10 \mathrm{~m}$ & $0.5 \mathrm{~s}$ & $0.05 \mathrm{~s}$ & 0.5 & $4 \Delta x$ \\
\hline Smagorinsky & $(43,43,43)$ & $64 \mathrm{~m}$ & $10 \mathrm{~m}$ & $1.0 \mathrm{~s}$ & $0.1 \mathrm{~s}$ & - & - \\
Smagorinsky & $(43,43,43)$ & $128 \mathrm{~m}$ & $10 \mathrm{~m}$ & $2.0 \mathrm{~s}$ & $0.2 \mathrm{~s}$ & - & - \\
Smagorinsky & $(43,43,83)$ & $32 \mathrm{~m}$ & $5 \mathrm{~m}$ & $0.5 \mathrm{~s}$ & $0.05 \mathrm{~s}$ & - & - \\
Smagorinsky & $(83,83,83)$ & $16 \mathrm{~m}$ & $5 \mathrm{~m}$ & $0.25 \mathrm{~s}$ & $0.025 \mathrm{~s}$ & - & - \\
Smagorinsky & $(83,83,83)$ & $32 \mathrm{~m}$ & $10 \mathrm{~m}$ & $0.5 \mathrm{~s}$ & $0.05 \mathrm{~s}$ & - & - \\
DRM-ADM0 & $(43,43,23)$ & $32 \mathrm{~m}$ & $20 \mathrm{~m}$ & $0.5 \mathrm{~s}$ & $0.05 \mathrm{~s}$ & 0.4 & $4 \Delta x$ \\
DRM-ADM0 & $(43,43,23)$ & $64 \mathrm{~m}$ & $20 \mathrm{~m}$ & $1.0 \mathrm{~s}$ & $0.1 \mathrm{~s}$ & 0.6 & $4 \Delta x$ \\
DRM-ADM0 & $(43,43,23)$ & $128 \mathrm{~m}$ & $20 \mathrm{~m}$ & $2.0 \mathrm{~s}$ & $0.2 \mathrm{~s}$ & 0.8 & $4 \Delta x$ \\
DRM-ADM0 & $(43,43,43)$ & $16 \mathrm{~m}$ & $16 \mathrm{~m}$ & $0.25 \mathrm{~s}$ & $0.025 \mathrm{~s}$ & 0.4 & $4 \Delta x$ \\
DRM-ADM0 & $(43,43,43)$ & $64 \mathrm{~m}$ & $10 \mathrm{~m}$ & $1.0 \mathrm{~s}$ & $0.1 \mathrm{~s}$ & 0.75 & $4 \Delta x$ \\
DRM-ADM0 & $(43,43,43)$ & $96 \mathrm{~m}$ & $10 \mathrm{~m}$ & $1.0 \mathrm{~s}$ & $0.1 \mathrm{~s}$ & 0.83 & $2 \Delta x$ \\
DRM-ADM0 & $(43,43,43)$ & $128 \mathrm{~m}$ & $10 \mathrm{~m}$ & $2.0 \mathrm{~s}$ & $0.2 \mathrm{~s}$ & 0.85 & $2 \Delta x$ \\
DRM-ADM0 & $(43,43,83)$ & $32 \mathrm{~m}$ & $5 \mathrm{~m}$ & $0.5 \mathrm{~s}$ & $0.05 \mathrm{~s}$ & 0.7 & $2 \Delta x$ \\
DRM-ADM0 & $(43,43,83)$ & $64 \mathrm{~m}$ & $5 \mathrm{~m}$ & $0.5 \mathrm{~s}$ & $0.05 \mathrm{~s}$ & 0.85 & $2 \Delta x$ \\
DRM-ADM0 & $(83,83,83)$ & $16 \mathrm{~m}$ & $5 \mathrm{~m}$ & $0.25 \mathrm{~s}$ & $0.025 \mathrm{~s}$ & 0.5 & $4 \Delta x$ \\
DRM-ADM0 & $(83,83,83)$ & $32 \mathrm{~m}$ & $10 \mathrm{~m}$ & $0.5 \mathrm{~s}$ & $0.05 \mathrm{~s}$ & 0.5 & $4 \Delta x$
\end{tabular}


Table 2: Computational cost of different turbulence models compared to the Smagorinsky model.

\begin{tabular}{lcc}
\hline \hline Model & Turbulence model cost factor & Total cost factor \\
\hline Smag & 1.00 & 1.00 \\
DWL (no near-wall) & 2.33 & 1.17 \\
DWL & 2.67 & 1.22 \\
DRM-ADM0 & 4.59 & 1.52 \\
DRM-ADM1 & 4.75 & 1.51 \\
DRM-ADM2 & 5.10 & 1.57 \\
DRM-ADM5 & 5.92 & 1.67 \\
DRM-ADM10 & 7.29 & 1.85
\end{tabular}




\section{List of Figures}

1 Schematic of velocity energy spectrum (with linear axes) showing partitioning into resolved, resolvable subfilter-scale (RSFS), and subgrid-scale (SGS) motions. The numerical error (NE) region is also shown above the dotted line. The grid is indicated by the vertical dashed line at wavenumber $k_{g}$ (corresponding to the minimum resolvable wavelength), and the explicit filter by the curved dashed line. . . . . . . 49

2 Comparison of non-stationarity parameters (a) $C_{u}$ and (b) $C_{v}$ for the Smagorinsky model and the DRM-ADM0 hybrid model. . . . . . . . . . . . . . . . 50

3 Comparison of (a) mean wind speed and (b) non-dimensional mean shear $\Phi$ profiles for no model, the Smagorinsky model, the dynamic Wong-Lilly model, and for the DRM-ADM0 hybrid model (level-0 reconstruction, DWL, and near-wall stress). Theoretical $\log$ profile also shown in (a). . . . . . . . . . . . . 51

4 Comparison of non-dimensional mean shear $\Phi$ profiles for the Smagorinsky model, also using the near-wall stress model, and DRM-ADM0 (with near-wall stress). . . 52

5 Comparison of non-dimensional mean shear $\Phi$ profiles for increasing reconstruction levels: dynamic Wong-Lilly, no reconstruction; DRM-ADM0; DRM-ADM1;

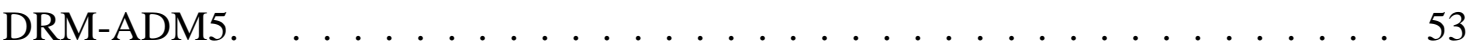

6 Comparison of $u w$ resolved (thin lines) and SFS (bold lines) stress profiles for Smagorinsky, DWL, and DRM-ADM0. . . . . . . . . . . . . . . 54

7 (a) Normalized vertical profiles of the $u w$ total stress for the DRM-ADM0 model (zero-level reconstruction, dynamic Wong-Lilly model, and near-wall canopy stress), with each model component shown separately in addition to the resolved stress. (b) Same as (a) but with a logarithmic vertical axis to magnify the region near the wall. 55

8 Comparison of $u w$ SGS (thin lines) and RSFS (bold lines) stress profiles for the dynamic Wong-Lilly SGS model with increasing RSFS reconstruction levels: DRMADM0; DRM-ADM1; DRM-ADM5. . . . . . . . . . . . 56 
9 Comparison of eddy-viscosity profiles for the dynamic Wong-Lilly SGS model with increasing RSFS reconstruction levels, shown for the bottom $200 \mathrm{~m}$ of the domain: DWL, no reconstruction; DRM-ADM0; DRM-ADM1; DRM-ADM5. . 57

10 Turbulent stresses computed a posteriori from velocity fields reconstructed from a fine resolution simulation (reconstructed from $(83,83,83)$ ), and the sum of RSFS and SGS stresses computed by coarse grid simulations $(43,43,43)$ : DWL, DRMADM0, DRM-ADM1, DRM-ADM2, DRM-ADM5, and DRM-ADM10. The nearwall stress contribution is not included. Averaged over 100000 to 120000 s. . . . 58

11 Comparison of non-dimensional mean shear $\Phi$ profiles for the Smagorinsky model and DRM-ADM0 for $43^{3}$ and $83^{3}$ grid sizes. Averaged from 75000 to 100000 s. . 59

12 Comparison of non-dimensional mean shear $\Phi$ profiles for (a) Smagorinsky and (b) DRM-ADM0 for different grid spacings or aspect ratios: $\Delta x: \Delta z_{\min }=32: 10$ (base case), 32:5, 64:10, 128:10. Note that the axes are scaled differently. Averaged from 75000 to $100000 \mathrm{~s} . \ldots \ldots \ldots$. . . . . . . . . . . 6 60

13 Variation of near-wall stress model proportionality constant with grid aspect ratio. A curve indicating the trend of the data is also shown. . . . . . . . . . 61 


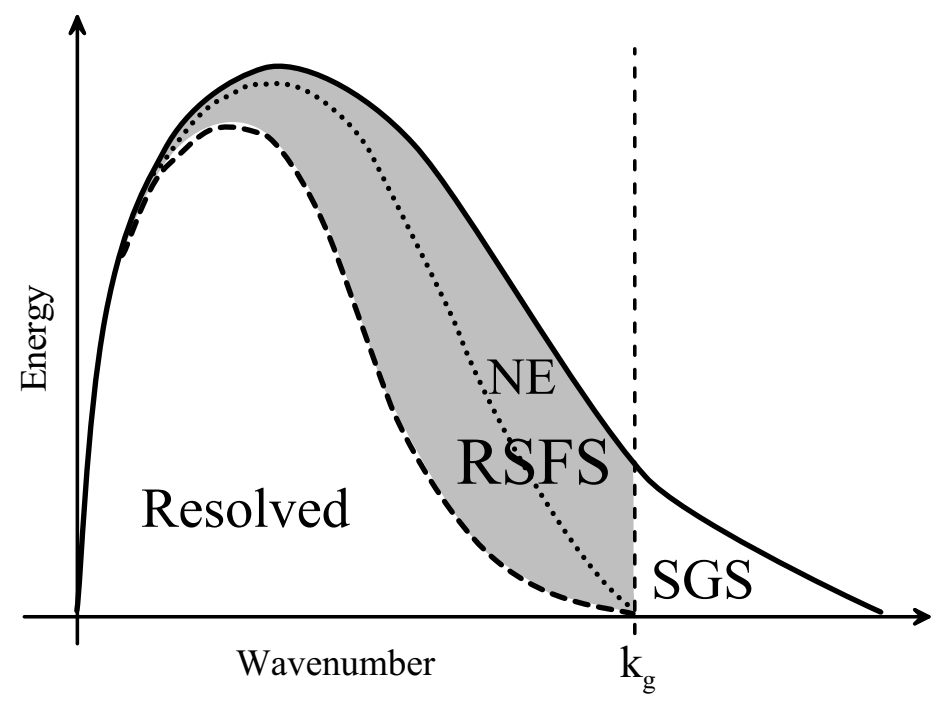

Figure 1: Schematic of velocity energy spectrum (with linear axes) showing partitioning into resolved, resolvable subfilter-scale (RSFS), and subgrid-scale (SGS) motions. The numerical error (NE) region is also shown above the dotted line. The grid is indicated by the vertical dashed line at wavenumber $k_{g}$ (corresponding to the minimum resolvable wavelength), and the explicit filter by the curved dashed line. 


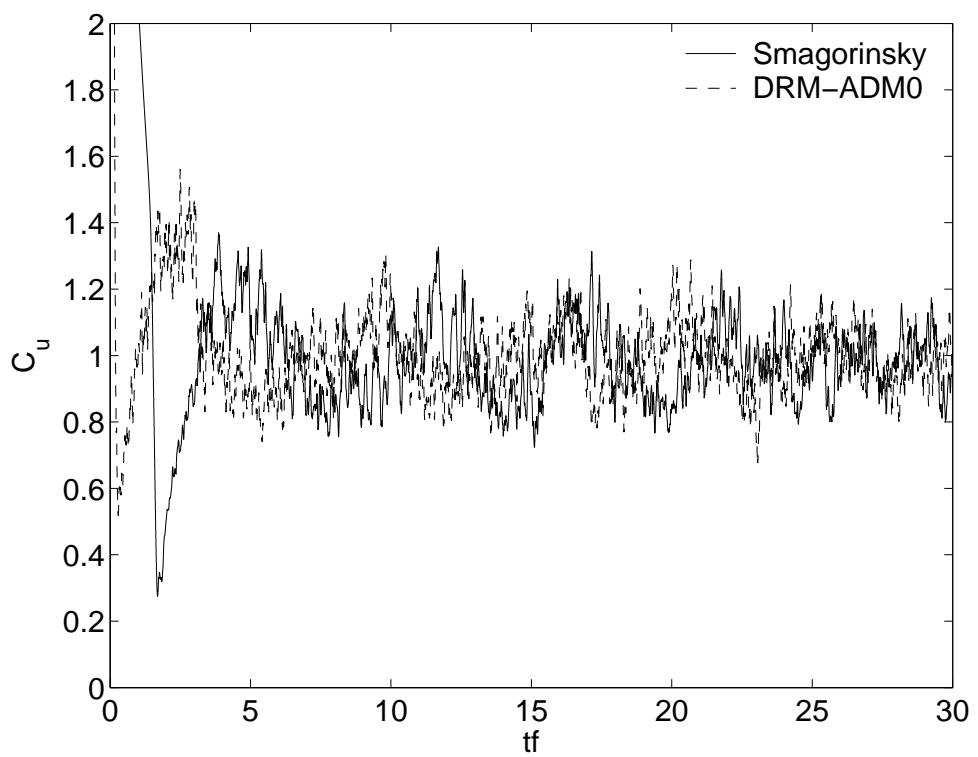

(a)

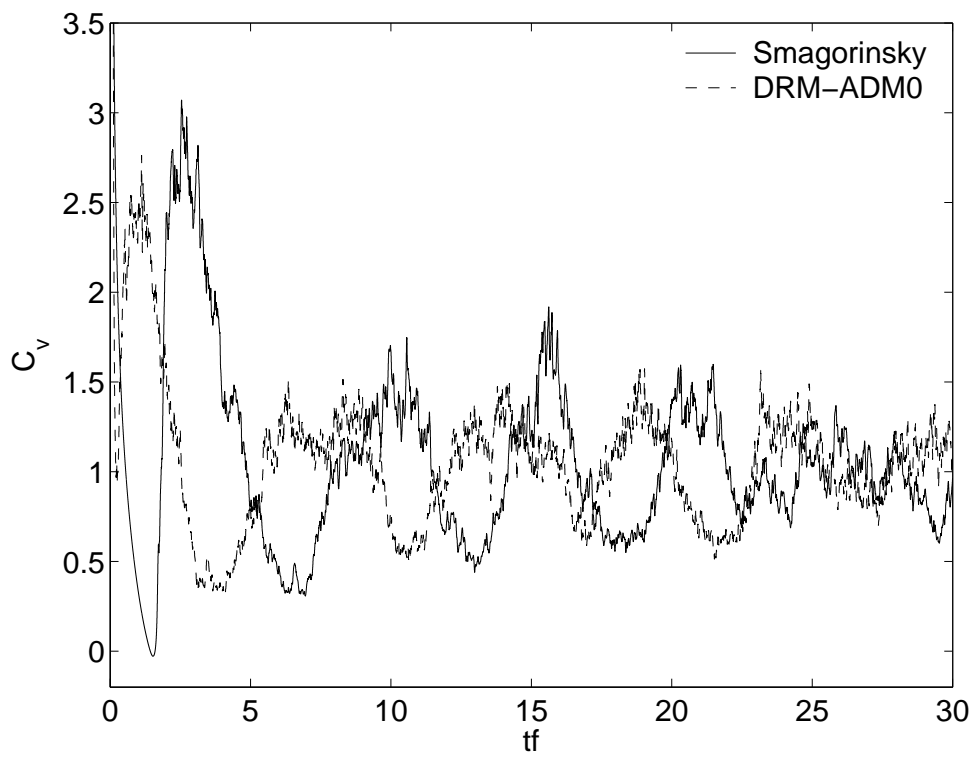

(b)

Figure 2: Comparison of non-stationarity parameters (a) $C_{u}$ and (b) $C_{v}$ for the Smagorinsky model and the DRM-ADM0 hybrid model. 


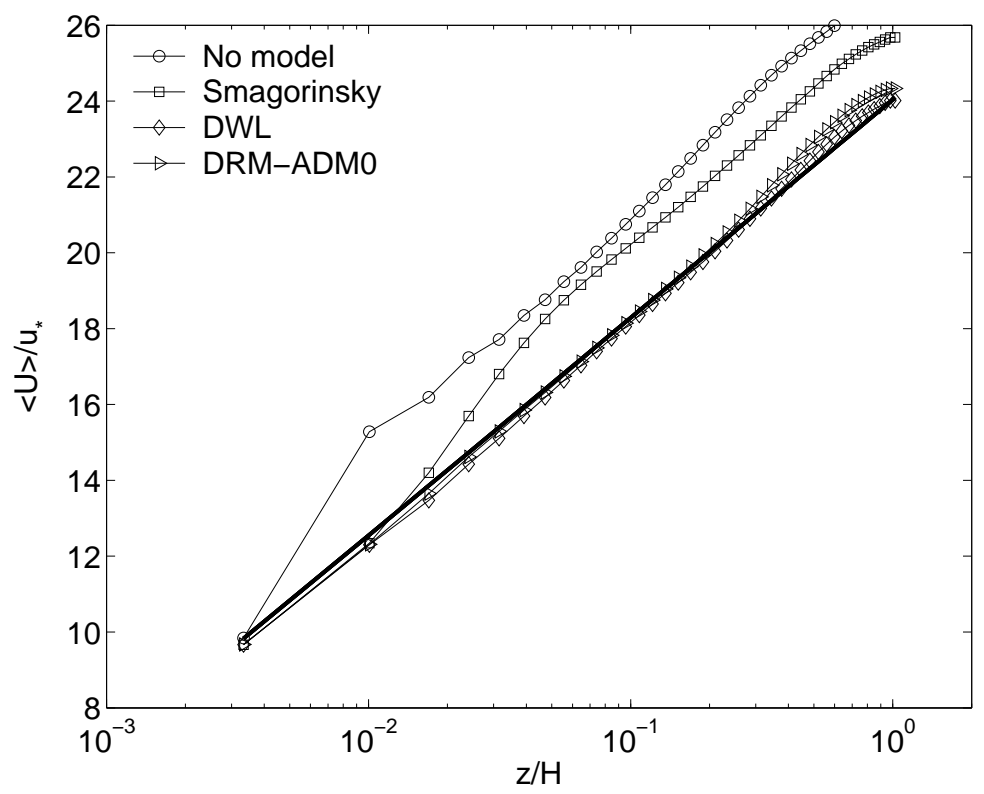

(a)

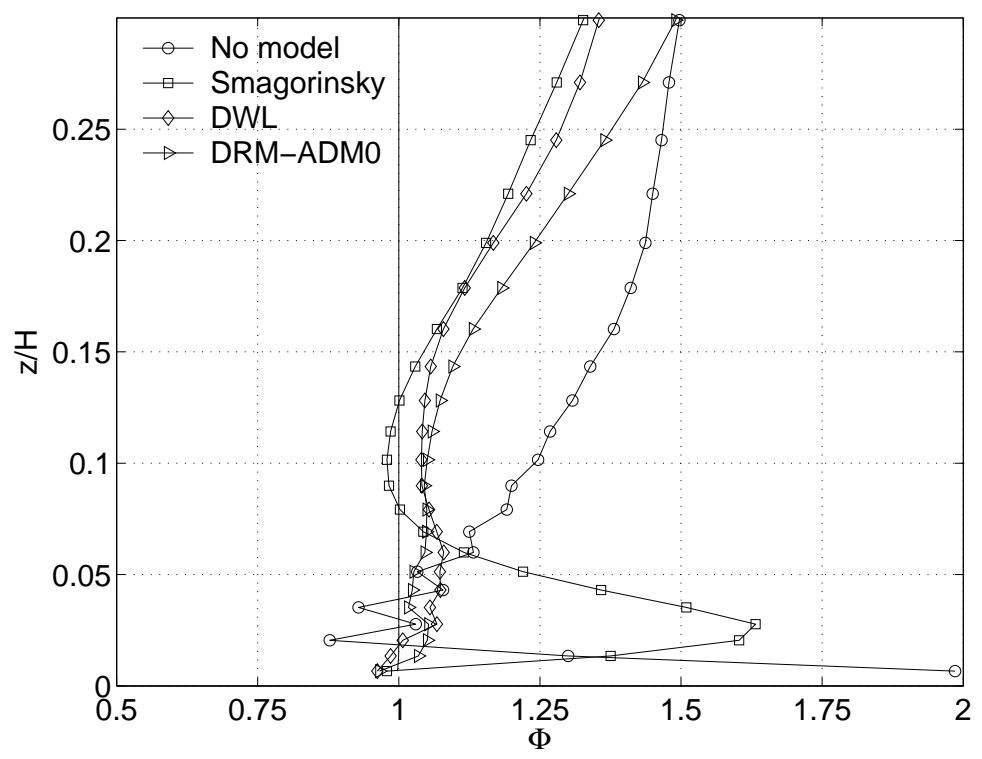

(b)

Figure 3: Comparison of (a) mean wind speed and (b) non-dimensional mean shear $\Phi$ profiles for no model, the Smagorinsky model, the dynamic Wong-Lilly model, and for the DRM-ADM0 hybrid model (level-0 reconstruction, DWL, and near-wall stress). Theoretical log profile also shown in (a). 


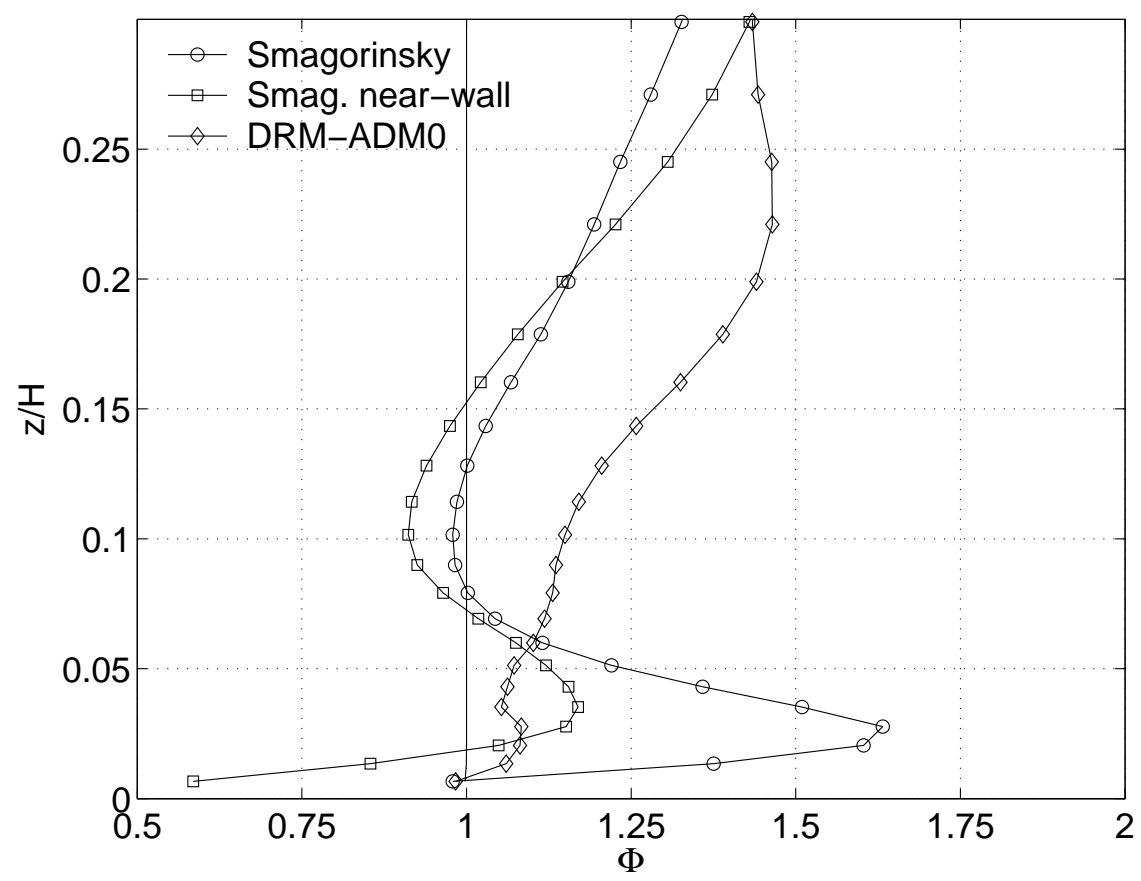

Figure 4: Comparison of non-dimensional mean shear $\Phi$ profiles for the Smagorinsky model, also using the near-wall stress model, and DRM-ADM0 (with near-wall stress). 


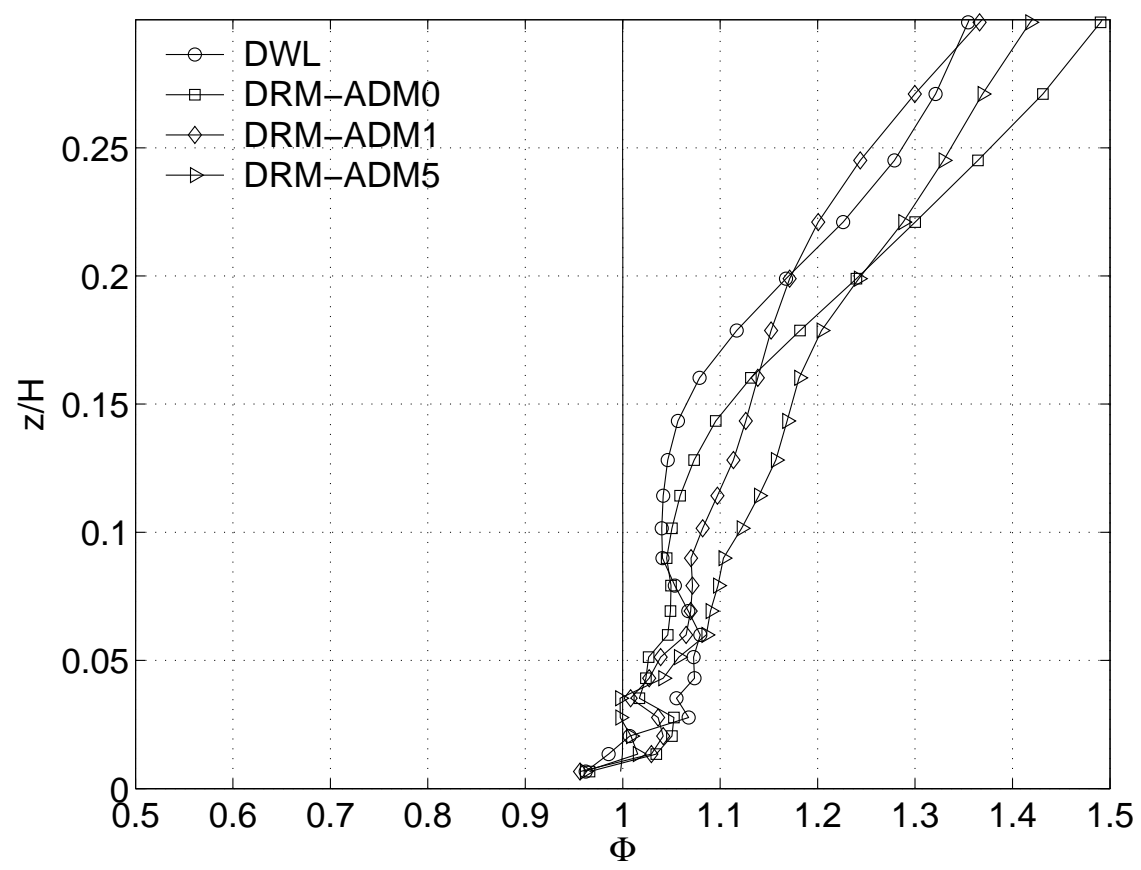

Figure 5: Comparison of non-dimensional mean shear $\Phi$ profiles for increasing reconstruction levels: dynamic Wong-Lilly, no reconstruction; DRM-ADM0; DRM-ADM1; DRM-ADM5. 


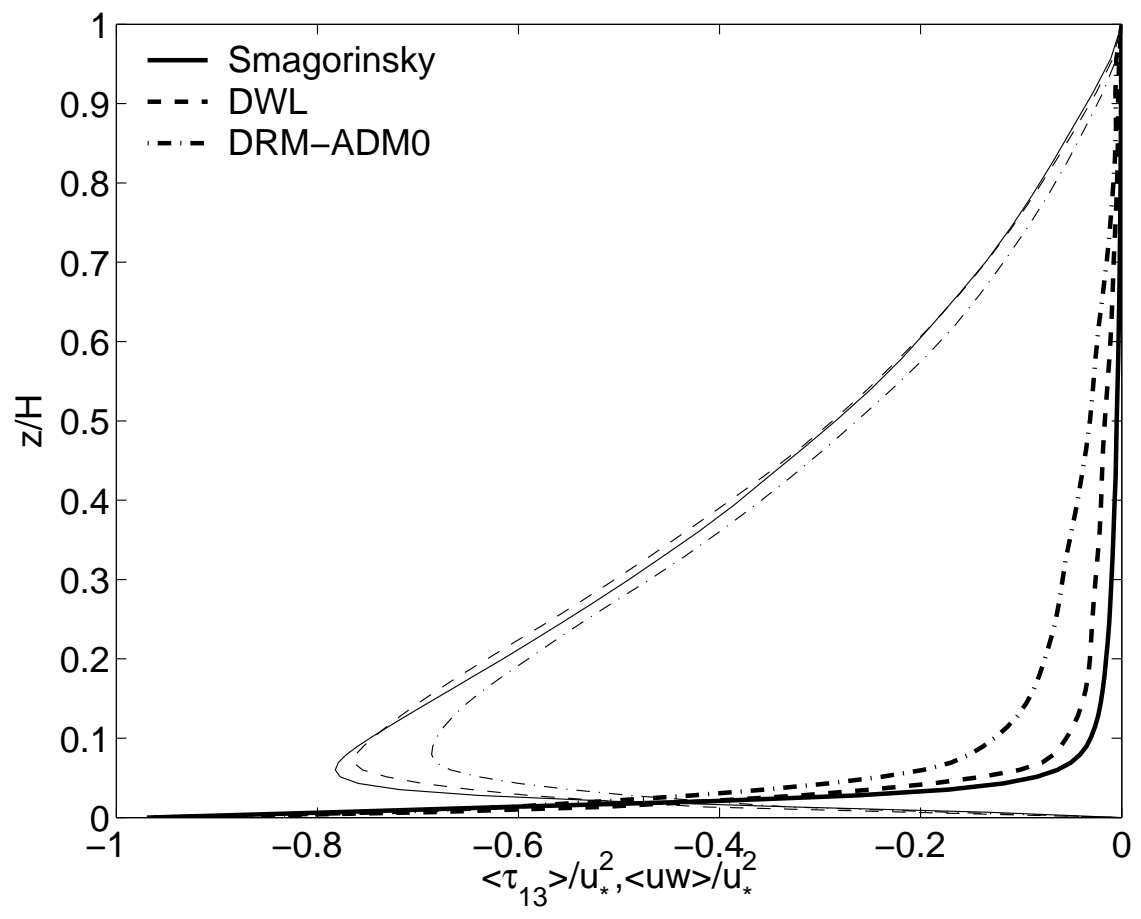

Figure 6: Comparison of $u w$ resolved (thin lines) and SFS (bold lines) stress profiles for Smagorinsky, DWL, and DRM-ADM0. 


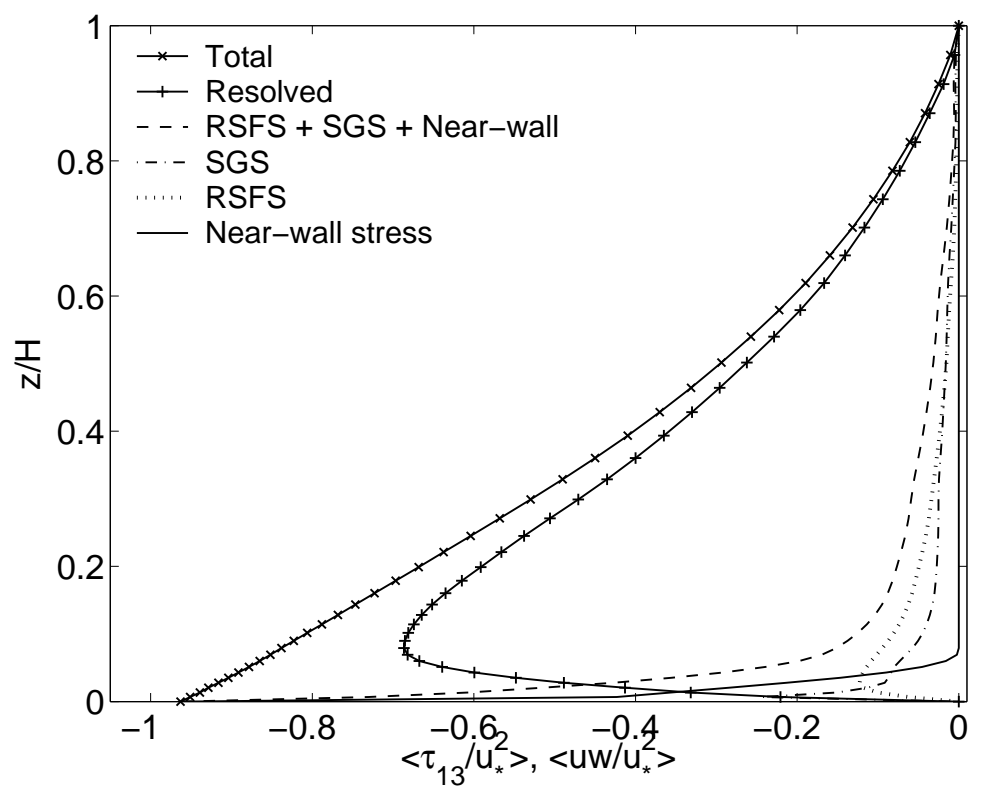

(a)

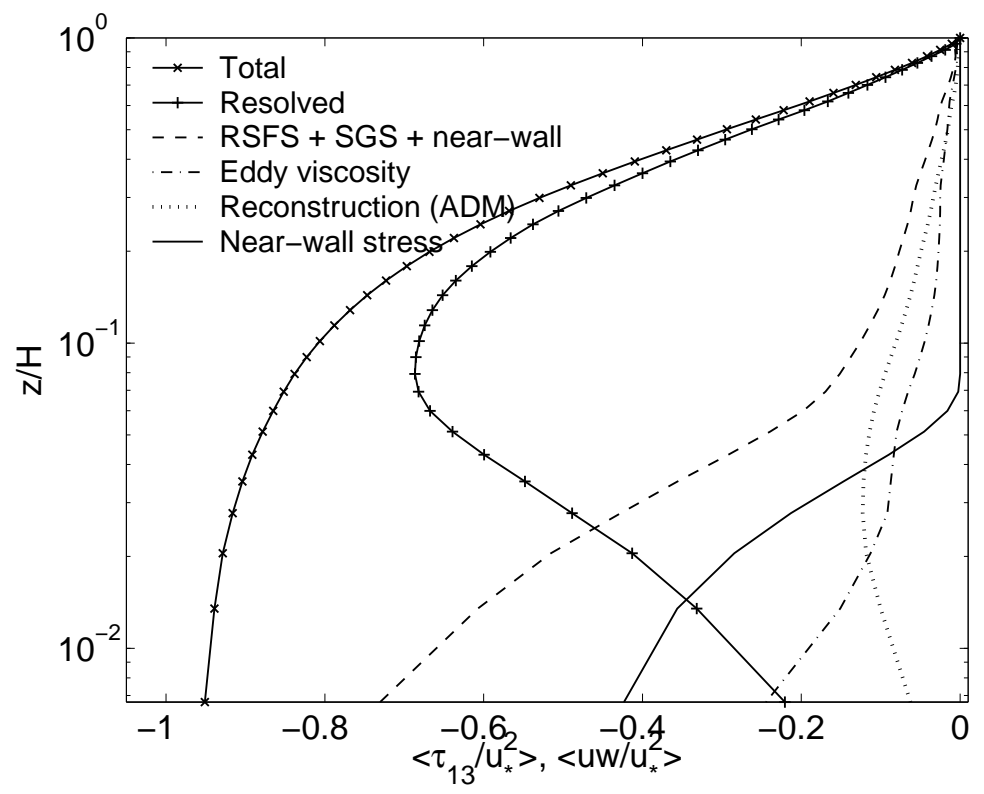

(b)

Figure 7: (a) Normalized vertical profiles of the $u w$ total stress for the DRM-ADM0 model (zerolevel reconstruction, dynamic Wong-Lilly model, and near-wall canopy stress), with each model component shown separately in addition to the resolved stress. (b) Same as (a) but with a logarithmic vertical axis to magnify the region near the wall. 


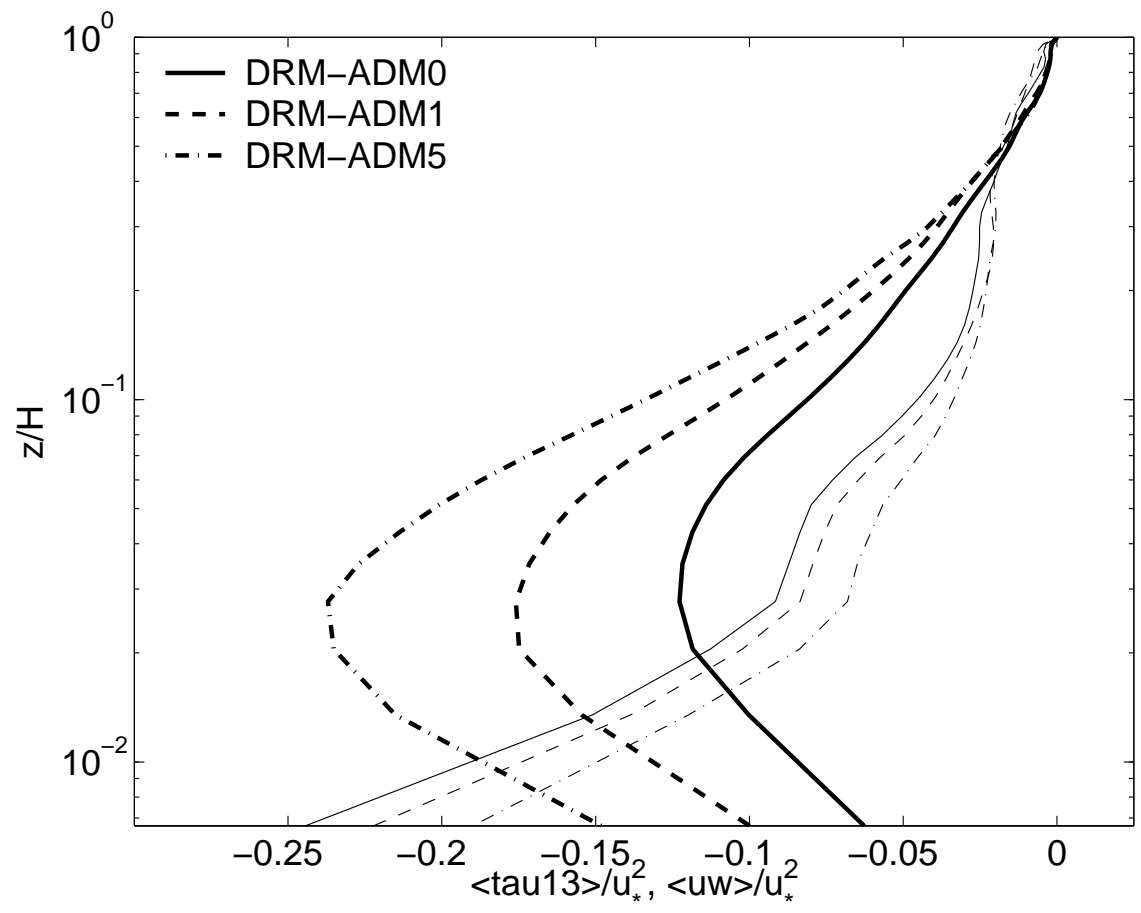

Figure 8: Comparison of $u w$ SGS (thin lines) and RSFS (bold lines) stress profiles for the dynamic Wong-Lilly SGS model with increasing RSFS reconstruction levels: DRM-ADM0; DRM-ADM1; DRM-ADM5. 


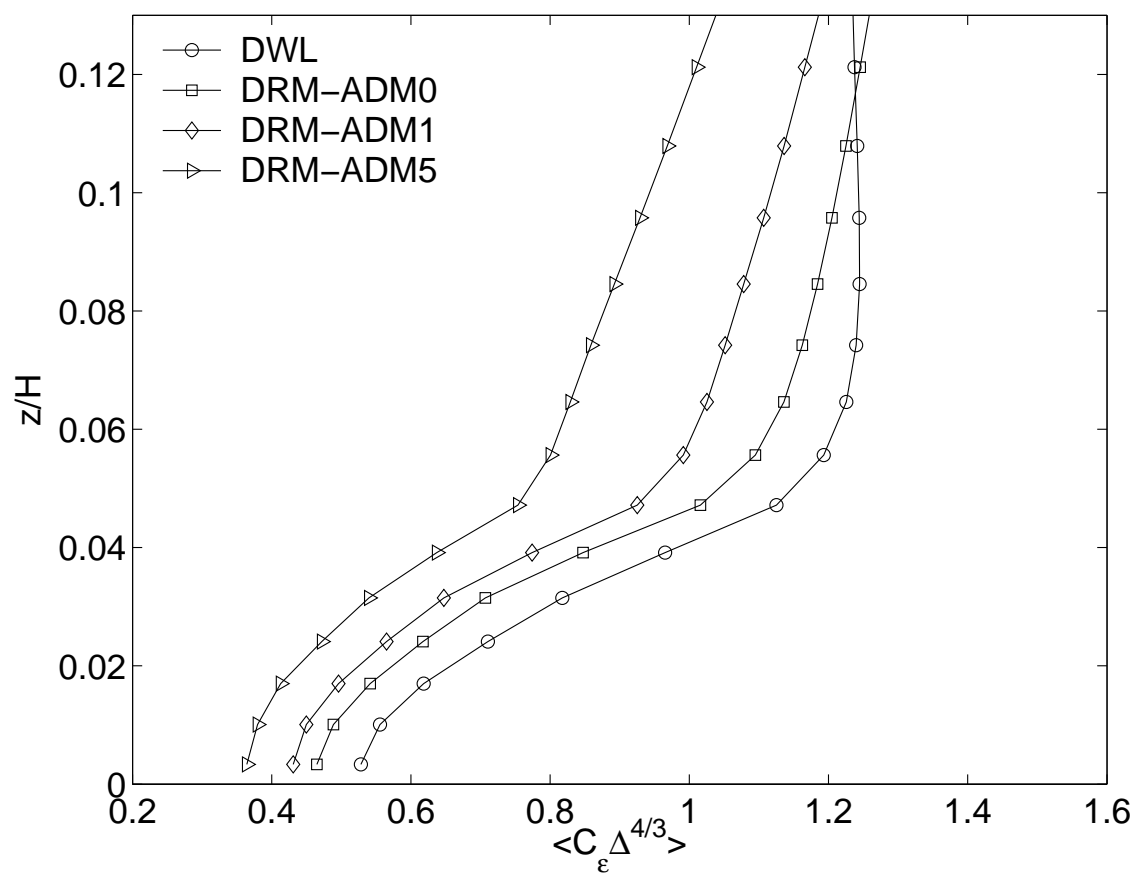

Figure 9: Comparison of eddy-viscosity profiles for the dynamic Wong-Lilly SGS model with increasing RSFS reconstruction levels, shown for the bottom $200 \mathrm{~m}$ of the domain: DWL, no reconstruction; DRM-ADM0; DRM-ADM1; DRM-ADM5. 


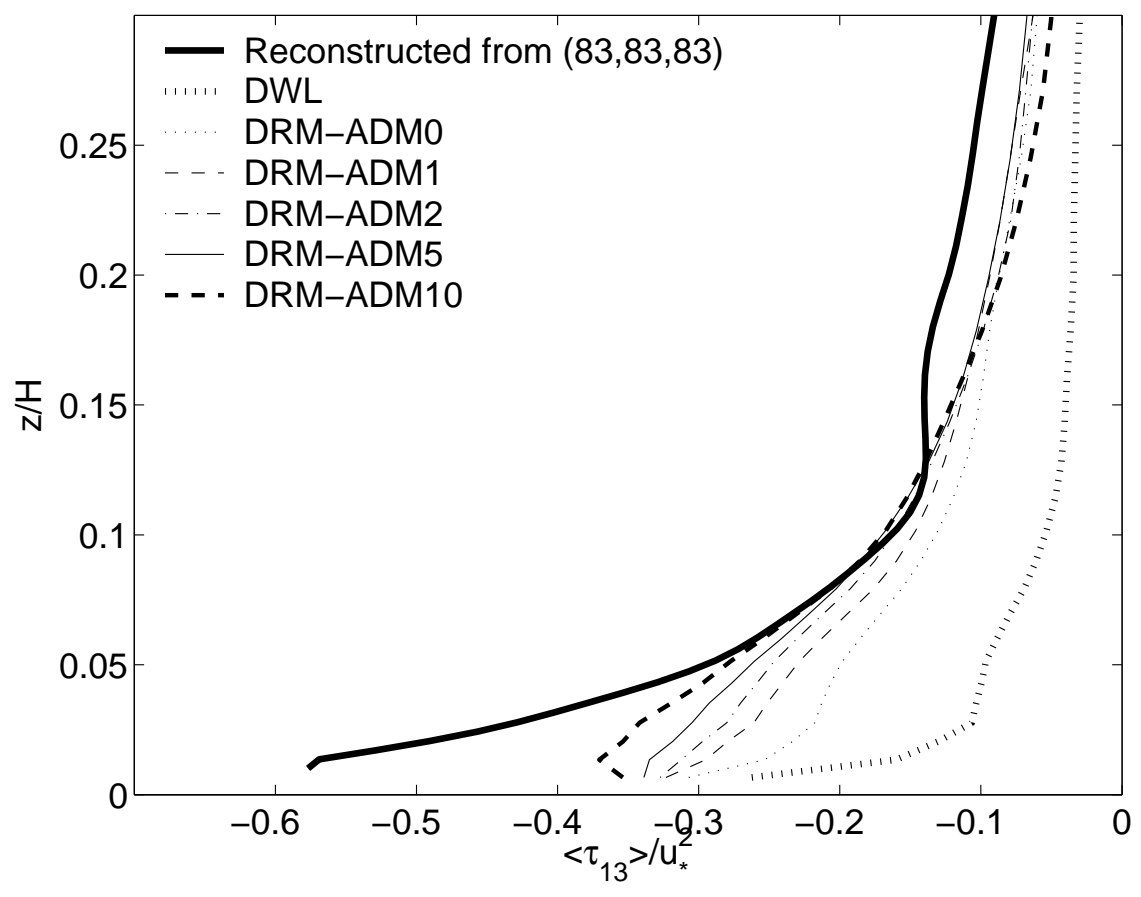

Figure 10: Turbulent stresses computed a posteriori from velocity fields reconstructed from a fine resolution simulation (reconstructed from $(83,83,83)$ ), and the sum of RSFS and SGS stresses computed by coarse grid simulations $(43,43,43)$ : DWL, DRM-ADM0, DRM-ADM1, DRM-ADM2, DRM-ADM5, and DRM-ADM10. The near-wall stress contribution is not included. Averaged over 100000 to $120000 \mathrm{~s}$. 


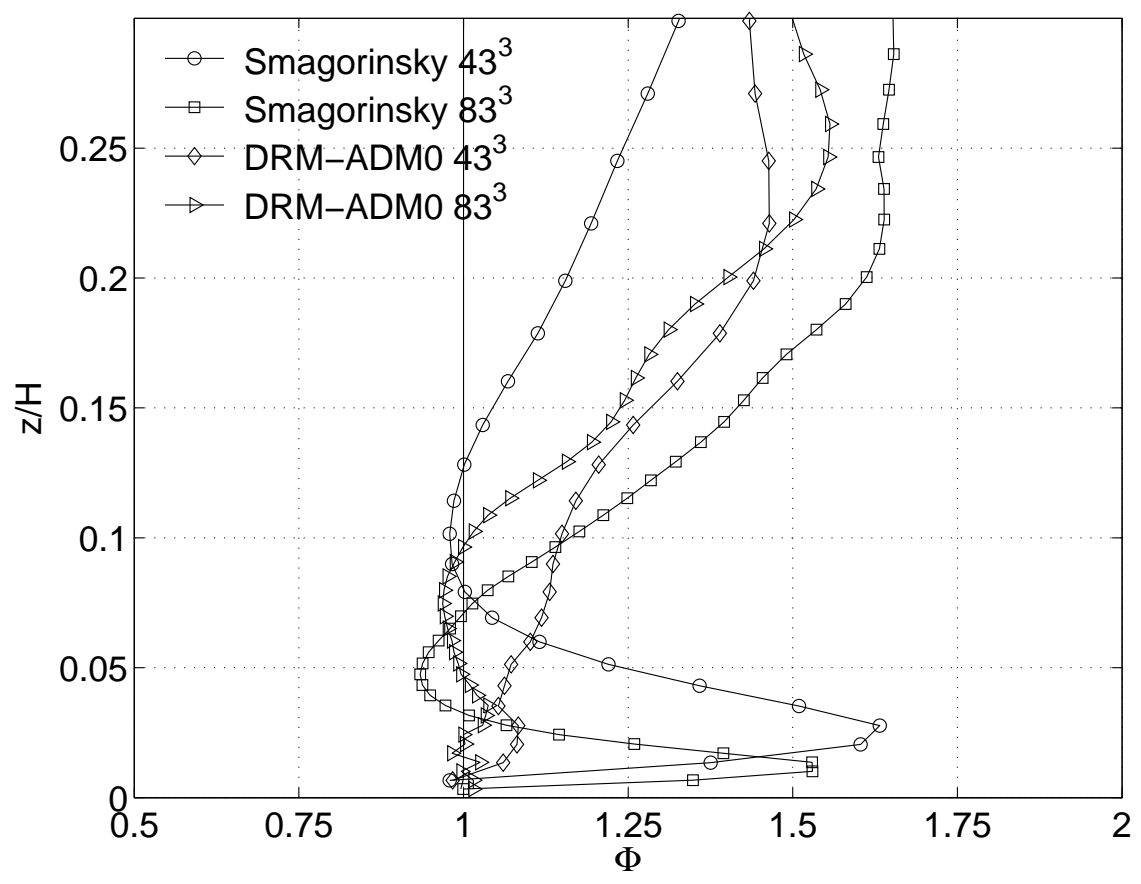

Figure 11: Comparison of non-dimensional mean shear $\Phi$ profiles for the Smagorinsky model and DRM-ADM0 for $43^{3}$ and $83^{3}$ grid sizes. Averaged from 75000 to $100000 \mathrm{~s}$. 


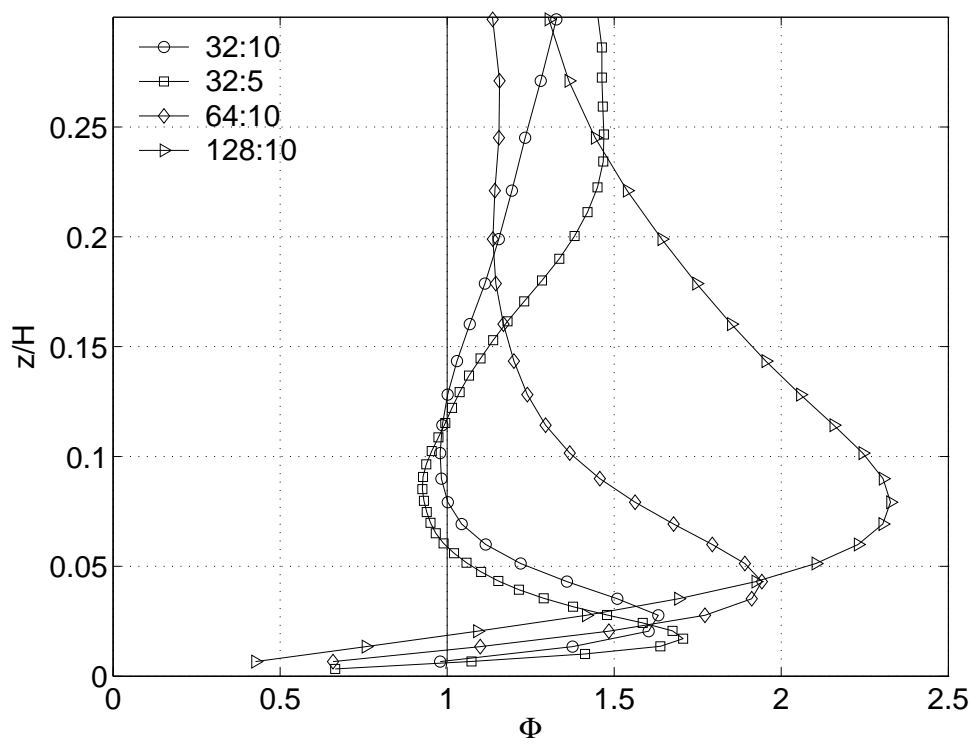

(a)

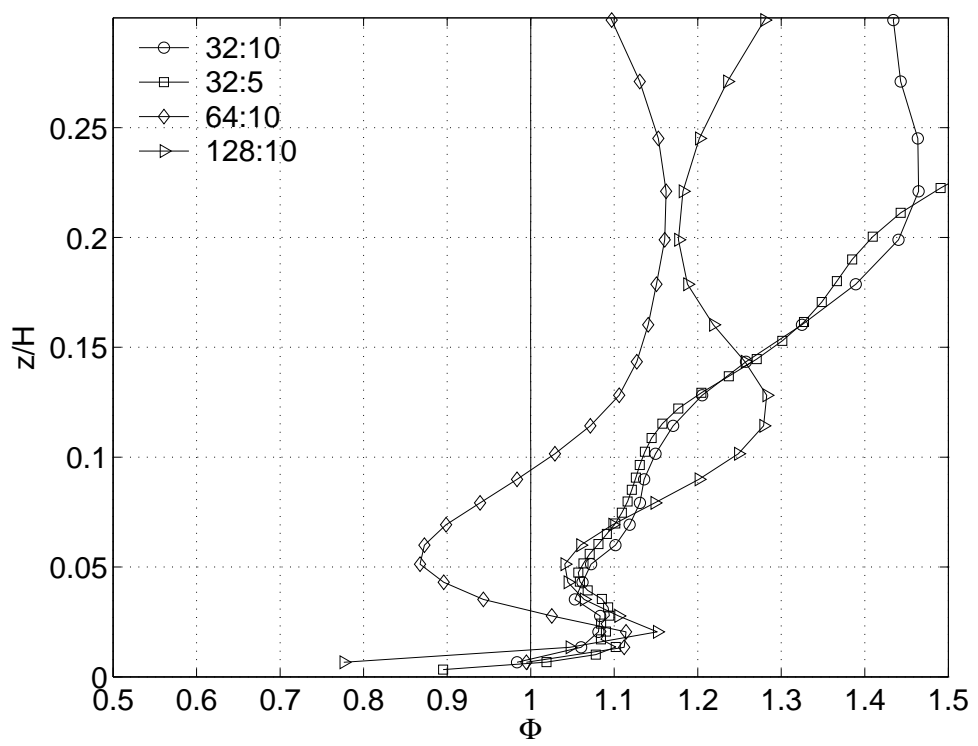

(b)

Figure 12: Comparison of non-dimensional mean shear $\Phi$ profiles for (a) Smagorinsky and (b) DRM-ADM0 for different grid spacings or aspect ratios: $\Delta x: \Delta z_{\min }=32: 10$ (base case), 32:5, 64:10, 128:10. Note that the axes are scaled differently. Averaged from 75000 to $100000 \mathrm{~s}$. 


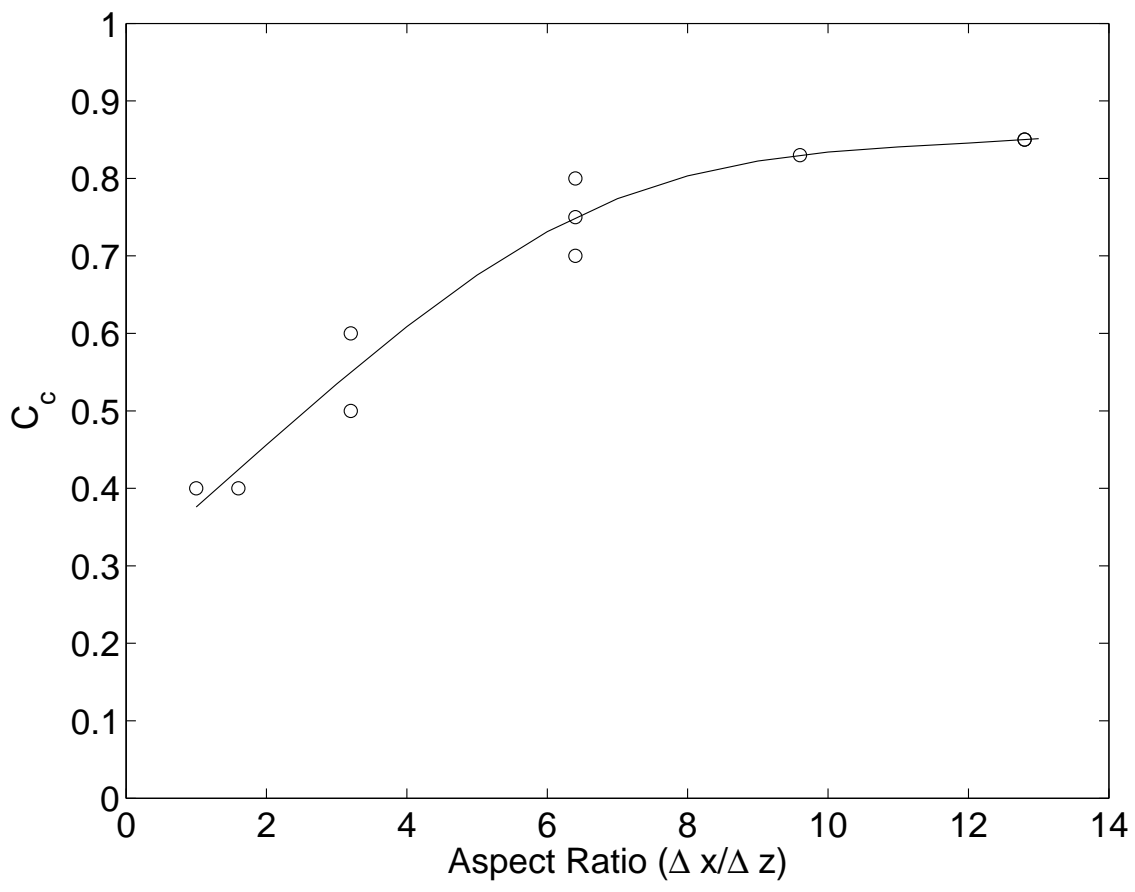

Figure 13: Variation of near-wall stress model proportionality constant with grid aspect ratio. A curve indicating the trend of the data is also shown. 